TRANSACTIONS OF THE

AMERICAN MATHEMATICAL SOCIETY

Volume 349, Number 2, February 1997, Pages 837-856

S 0002-9947(97)01748-0

\title{
CONGRUENCES BETWEEN MODULAR FORMS, CYCLIC ISOGENIES OF MODULAR ELLIPTIC CURVES, AND INTEGRALITY OF $p$-ADIC $L$-FUNCTIONS
}

\author{
SHU-LEUNG TANG
}

\begin{abstract}
Let $\Gamma$ be a congruence subgroup of type $\left(N_{1}, N_{2}\right)$ and of level $N$. We study congruences between weight 2 normalized newforms $f$ and Eisenstein series $E$ on $\Gamma$ modulo a prime $\mathfrak{p}$ above a rational prime $p$. Assume that $p \nmid 6 N$, $E$ is a common eigenfunction for all Hecke operators and $f$ is ordinary at $\mathfrak{p}$. We show that the abelian variety associated to $f$ and the cuspidal subgroup associated to $E$ intersect non-trivially in their $p$-torsion points. Let $A$ be a modular elliptic curve over $\mathbb{Q}$ with good ordinary reduction at $p$. We apply the above result to show that an isogeny of degree divisible by $p$ from the optimal curve $A_{1}$ in the $\mathbb{Q}$-isogeny class of elliptic curves containing $A$ to $A$ extends to an étale morphism of Néron models over $\mathbb{Z}_{p}$ if $p>7$. We use this to show that $p$-adic distributions associated to the $p$-adic $L$-functions of $A$ are $\mathbb{Z}_{p}$-valued.
\end{abstract}

\section{INTRODUCTION}

Let $A$ be a modular elliptic curve of conductor $N$ defined over $\mathbb{Q}$ and let $p>2$ be a prime at which $A$ has good ordinary reduction. Let $\Delta$ be a positive integer prime to $p$. In [9], Mazur and Swinnerton-Dyer construct, using modular symbols, an $H_{1}(A, \mathbb{Z}) \otimes \mathbb{Q}_{p}$-valued measure $\mu_{A, \Delta}$ on $\mathbb{Z}_{p, \Delta}^{*}=\longleftarrow \lim \left(\mathbb{Z} / p^{n} \Delta \mathbb{Z}\right)^{*}$ (inverse limit being with respect to natural maps) associated to $\overleftarrow{A}$, and define the $p$-adic $L$ function of $A$ to be the $p$-adic Mellin transform of $\mu_{A, \Delta}$ which interpolates the values of the complex $L$-functions $L(A, \chi, z)$ at $z=1$ for Dirichlet characters $\chi$ of conductor $p^{n} \Delta, n \geq 0$. In the light of Iwasawa theory, one would expect $\mu_{A, \Delta}$ to be $H_{1}(A, \mathbb{Z}) \otimes \mathbb{Z}_{p}$-valued. This is known when $\Delta=1$.

Let $\pi: X_{0}(N) \rightarrow A$ be a modular parametrization (i.e. a non-constant $\mathbb{Q}$ morphism) which sends the cusp $\infty$ to the origin of $A$. Let $\omega_{A}$ be a Néron differential on $A$. Then $\pi^{*} \omega_{A}=c(\pi) f(q) d q / q$, where $c(\pi) \in \mathbb{Q}^{*}$ and $f(q) d q / q$ is a normalized newform on $\Gamma_{0}(N) . c(\pi)$ is called the Manin constant of $\pi$ and is conjectured by Manin to be \pm 1 when $A$ is strong Weil [8]. Stevens in [23] studies parametrizations $\pi: X_{1}(N) \rightarrow A$ (which send the cusp $0\left(=\left[\begin{array}{l}0 \\ 1\end{array}\right]\right)$ to the origin) and refines Manin's conjecture as follows.

Conjecture 0.1. ([23, Conj. I]) For every modular elliptic curve $A$ over $\mathbb{Q}$, there is a modular parametrization $X_{1}(N) \rightarrow A$ whose Manin constant is \pm 1 .

Received by the editors May 10, 1995 and, in revised form, September 21, 1995.

1991 Mathematics Subject Classification. Primary 11G05, 11G18; Secondary 11S40.

Key words and phrases. Modular forms, elliptic curves, $p$-adic $L$-functions. 
In [12], $p$-adic distributions on $\mathbb{Z}_{p, \Delta}^{*}$ are constructed more generally for modular forms of weight $\geq 2$. In the case of $f$ arising from $A$ as above, the construction yields an $\mathcal{L}(A) \otimes \mathbb{Q}_{p}$-valued measure $\nu_{A, \Delta}$ where $\mathcal{L}(A)$ is the period lattice of $A$ with respect to $\omega_{A}$ (cf. [23, §4]). If $\Delta=1, \mu_{A, \Delta}$ and $\nu_{A, \Delta}$ coincide (up to a $p$-adic unit) under the identification $H_{1}(A, \mathbb{Z}) \cong \mathcal{L}(A)$ but may differ in general (due to the $p$-adic "multiplier" $[12, \S 14])$. It is known $([23$, Thms. 1.6, 4.6]) that $c(\pi) \in \mathbb{Z}$ and $c(\pi) \nu_{A, \Delta}$ is $\mathcal{L}(A) \otimes \mathbb{Z}_{p}$-valued for any modular parametrization $\pi: X_{1}(N) \rightarrow A$. Thus if Conjecture 0.1 is true, then $\nu_{A, \Delta}$ is $\mathcal{L}(A) \otimes \mathbb{Z}_{p}$-valued. We study in this paper the integrality of $\nu_{A, \Delta}$ and prove the following (cf. [23, §4, Conj. IV])

Theorem 0.2. With the above notation and assumptions, $\nu_{A, \Delta}$ is $\mathcal{L}(A) \otimes \mathbb{Z}_{p}$-valued for $p>7$.

To prove Theorem 0.2 , it suffices to show $p \nmid c(\pi)$ for some modular parametrization $\pi: X_{1}(N) \rightarrow A$. Let $\mathcal{A}$ be the $\mathbb{Q}$-isogeny class of elliptic curves over $\mathbb{Q}$ containing $A$. Then there are a unique curve (up to $\mathbb{Q}$-isomorphism) $A_{1}$ in $\mathcal{A}$ and a modular parametrization $\pi_{1}: X_{1}(N) \rightarrow A_{1}$ such that if $\pi: X_{1}(N) \rightarrow A^{\prime}$ is a modular parametrization of a curve $A^{\prime} \in \mathcal{A}$, then there is a $\mathbb{Q}$-isogeny $\beta: A_{1} \rightarrow A^{\prime}$ such that $\pi=\beta \circ \pi_{1}$. We call $A_{1}$ the optimal curve in $\mathcal{A}$ and $\pi_{1}$ an optimal parametrization. By a result analogous to [11, Cor. 4.1], $c\left(\pi_{1}\right) \in \mathbb{Z}[1 / 2 n]^{*}$ where $n$ is the largest square dividing $N$. (Mazur proves this for $X_{0}(N)$-parametrizations of strong Weil curves, but his method works also for $X_{1}(N)$-parametrizations of optimal curves.) In particular, $p \nmid c\left(\pi_{1}\right)$. The next step is to look at $\mathbb{Q}$-isogenies between $A_{1}$ and $A$. In this direction, we prove

Theorem 0.3. Suppose $p>7$. Let $\beta: A_{1} \rightarrow A$ be a cyclic $\mathbb{Q}$-isogeny of degree divisible by $p$. Then $\beta$ is étale at $p$.

Here we say that an isogeny $\alpha: E_{1} \rightarrow E_{2}$ of elliptic curves over $\mathbb{Q}$ is étale at $p$ if the morphism $\alpha_{/ \mathbb{Z}_{p}}: E_{1 / \mathbb{Z}_{p}} \rightarrow E_{2 / \mathbb{Z}_{p}}$ of Néron models over $\mathbb{Z}_{p}$ is étale. The proof of Theorem 0.3 relies on Theorem 0.4 below, which reflects a general principle in the theory of modular curves that whenever there is a congruence between two modular forms, there should be a fusion module which explains the congruence. Let $\Gamma$ be a congruence group of type $\left(N_{1}, N_{2}\right)$ and of level $N([21,1.1])$. Let $f$ (resp. $E$ ) be a weight two normalized newform (resp. an Eisenstein series) on $\Gamma$. Assume that $E$ is a common eigenfunction for all Hecke operators and that there is a place $\mathfrak{P}$ of $\overline{\mathbb{Q}}$ such that the Fourier coefficients of $f$ and $E$ are congruent mod $\mathfrak{P}$. We say that $\mathfrak{P}$ is an Eisenstein prime for $E$ and $f$. Let $A_{f}$ be the abelian subvariety over $\mathbb{Q}$ of the Jacobian $J_{\Gamma}$ of the modular curve associated to $\Gamma$ ([18, Thm. 7.14]) and $K_{f}$ the field generated over $\mathbb{Q}$ by the Fourier coefficients of $f$. There is an embedding of $K_{f}$ into $\operatorname{End}\left(A_{f}\right) \otimes_{\mathbb{Z}} \mathbb{Q}$ (loc. cit.). Let $C_{E}$ be the cuspidal subgroup of $J_{\Gamma}$ associated to $E([21,1.8])$. Let $\mathfrak{p}$ be the prime of $K_{f}$ below $\mathfrak{P}$. We say that $f$ is ordinary at $\mathfrak{p}$ if the $p$-th Fourier coefficient of $f$ is a unit mod $\mathfrak{p}$. By considering the $q$-expansions in characteristic $p$ of $A_{f}[\mathfrak{p}]$ and $C_{E}[p]$, we prove

Theorem 0.4. Let $\mathfrak{P}$ be an Eisenstein prime for $f$ and $E$. Assume that $\mathfrak{P} \nmid 6 N$ and $f$ is ordinary at $\mathfrak{p}$. Then $A_{f}[\mathfrak{p}] \cap C_{E} \neq 0$.

Returning to the proof of Theorem 0.3 , we let $\beta: A_{1} \rightarrow A$ be a cyclic isogeny of degree divisible by $p$. Assuming that the Galois character on the subgroup of order $p$ of ker $\beta$ is not the trivial character or the Teichmüller character, we show that there is an Eisenstein series $E$ on $\Gamma_{1}(N)$ arising from the Galois representation on 
the $p$-torsion points of $A_{1}$ whose Fourier coefficients are congruent mod $\mathfrak{P}$ to those of $f$ (Prop. 2.6). Theorem 0.4 shows that $A_{1}$ and the cuspidal subgroup of the Jacobian of $X_{1}(N)$ associated to $E$ intersect non-trivially in their $p$-torsion points. Using the classification theorem of rational cyclic isogenies of elliptic curves over $\mathbb{Q}$ ([11], [7]), we deduce that $p \leq 7(\S 2.3)$.

We prove Theorems 0.4, 0.3 and 0.2 in Sections 1, 2 and 3 respectively . For a field $K$, we write $\bar{K}$ for an algebraic closure of $K, K_{s}$ for the separable closure of $K$ in $\bar{K}$ and $G_{K}$ for $\operatorname{Gal}\left(K_{s} / K\right)$. Throughout the paper, we fix a place $\mathfrak{P}$ of $\overline{\mathbb{Q}}$ above $p$ and identify the residue field of the valuation ring of $\overline{\mathbb{Q}}$ at $\mathfrak{P}$ as an algebraic closure $\overline{\mathbb{F}}_{p}$ of $\mathbb{F}_{p}$. Also fix an embedding of $\overline{\mathbb{Q}}$ into $\mathbb{C}_{p}$ so that $\mathfrak{P}$ is the place induced by it. For a prime $l, D_{l}$ will denote a decomposition group in $G_{\mathbb{Q}}$ for $l$ and $I_{l}$ its inertia subgroup. For a commutative group scheme $G$ and $\alpha$ an endomorphism of $G, G[\alpha]$ will denote the kernel of $\alpha$ and $G\left[\alpha^{\infty}\right]=\bigcup_{n \geq 0} G\left[\alpha^{n}\right]$. If $\mathfrak{a}$ is a set of endomorphisms of $G$, then $G[\mathfrak{a}]$ will denote $\bigcap_{\alpha \in \mathfrak{a}} G[\alpha]$. When $G=\mathbb{G}_{m}$ is the multiplicative group, we write $\mu_{n}$ for $\mathbb{G}_{m}[n]$. We shall use the following notation for cusps: if $\Gamma$ is a congruence subgroup of level $N$ and $a, b \in \mathbb{Z}$ are such that $(a, b, N)=1$, then $\left[\begin{array}{l}a \\ b\end{array}\right]=\left[\begin{array}{l}a \\ b\end{array}\right]_{\Gamma}$ will denote the $\Gamma$-equivalence class of the cusp $a / b$.

Acknowledgments. This work was done while the author was a post-doctoral fellow at McMaster University. Their support is gratefully acknowledged. I also thank the referee for suggestions which led to an improvement of Theorem 0.4.

\section{Fusion module of an Eisenstein prime}

In this section, we prove Theorem 0.4. Let $\Gamma$ be a congruence subgroup of type $\left(N_{1}, N_{2}\right)$ and of level $N$. Let $f$ (resp. $E$ ) be a weight two normalized newform (resp. an Eisenstein series) on $\Gamma$. Suppose that $E$ is a common eigenfunction for all Hecke operators. Let $a_{n}(n \geq 0)$ (resp. $\left.b_{n}(n \geq 0)\right)$ be the Fourier coefficients of $f$ (resp. $E$ ). (We take $a_{0}=0$.) Assume that $\mathfrak{P}$ is an Eisenstein prime associated to $f$ and $E$, i.e. $a_{n} \equiv b_{n}(\bmod \mathfrak{P})$ for all $n \geq 0$. We write $f \equiv E(\bmod \mathfrak{P})$. Assume further that $\mathfrak{P} \nmid 6 N$ and $f$ is ordinary at $\mathfrak{p}$. We first review some properties of regular differentials on modular curves and their $q$-expansions $(\S 1.2)$ and the $q$-expansions of the $p$-torsion points of the Jacobian of a curve over $\overline{\mathbb{F}}_{p}(\S 1.3)$. We show that the $q$-expansions in characteristic $p$ of $A_{f}[\mathfrak{p}]$ and the cuspidal subgroup $C_{E}[p]$ coincide. This enables us to conclude that $A_{f}[\mathfrak{p}]$ and $C_{E}$ intersect non-trivially.

1.1. Modular curves and Hecke operators. Let $\mathfrak{H}$ be the upper half plane and $\mathfrak{H}^{*}=\mathfrak{H} \cup \mathbb{P}^{1}(\mathbb{Q})$. The quotient $\mathfrak{H} / \Gamma$ is an open Riemann surface which can be compactified to a projective algebraic curve $X_{\Gamma / \mathbb{C}}=\mathfrak{H}^{*} / \Gamma$ over $\mathbb{C}$ by the addition of cusps. By Shimura $[18, \S 6.7], X_{\Gamma / \mathbb{C}}$ has a canonical model $X_{\Gamma}$ over $\mathbb{Q}$. The moduli interpretation of $X_{\Gamma}$ is that it is the coarse moduli scheme of the functor associating to each $\mathbb{Q}$-scheme $S$ the $S$-isomorphism classes of generalized elliptic curves over $S$ with an $H$-orbit of level $N$-structures where $H$ is the image of $\Gamma$ under the natural map $\Gamma \rightarrow \mathrm{GL}_{2}(\mathbb{Z} / N \mathbb{Z})$. Write $X(N), X_{0}(N), X_{1}(N)$ for $\Gamma=\Gamma(N), \Gamma_{0}(N), \Gamma_{1}(N)$ respectively.

Let $T_{l}(l \nmid N)$ and $U_{l}(l \mid N)$ be the usual Hecke correspondences on $X_{\Gamma}$ (see for example [18, Chap. 7]). For $m \in(\mathbb{Z} / N \mathbb{Z})^{*}$, let $\sigma_{m} \in \mathrm{SL}_{2}(\mathbb{Z})$ be such that

$$
\sigma_{m} \equiv\left(\begin{array}{cc}
* & 0 \\
0 & m
\end{array}\right) \quad(\bmod N)
$$


and write $\langle m\rangle$ for the Hecke correspondence corresponding to $\sigma_{m}$. Let $J_{\Gamma}$ be the Jacobian of $X_{\Gamma}$ and $\mathbb{T} \subset \operatorname{End}\left(J_{\Gamma}\right) \otimes_{\mathbb{Z}} \mathbb{Q}$ the Hecke algebra generated by the images of $T_{l}(l \nmid N), U_{l}(l \mid N)$ and $\langle m\rangle, m \in(\mathbb{Z} / N \mathbb{Z})^{*}$. Denote the images of $T_{l}, U_{l}$ and $\langle m\rangle$ in $\mathbb{T}$ by the same symbols. For brevity, we shall sometimes write $T_{l}$ for $U_{l}$ when $l \mid N$ below.

1.2. Regular differentials and their $q$-expansions. Let $\zeta_{N}$ be a primitive $N$-th root of unity. Let $\mathcal{O}$ be the completion of $\mathbb{Z}\left[\zeta_{N}\right]$ at the prime below $\mathfrak{P}$, and $K$ the field of fractions of $\mathcal{O}$. Fix an embedding of $K$ in $\mathbb{C}$. Let $X_{\Gamma / \mathcal{O}}$ be the normalization of the $j$-line $\mathbb{P}_{/ \mathcal{O}}^{1}$ in the function field of $Y_{\Gamma / K}$, where the morphism $Y_{\Gamma / K} \rightarrow \mathbb{P}^{1} \mathcal{O}$ is defined on points by sending an elliptic curve $E$ with level $H$-structure to the $j$-invariant of $E$. Then $X_{\Gamma / \mathcal{O}}$ is smooth. For any ring $R$ over $\mathcal{O}$, let $\Omega_{/ R}=\Omega_{X_{\Gamma} / R}$ be the sheaf of regular differentials with respect to $X_{\Gamma / R} \rightarrow \operatorname{Spec} R$ ([5, I 2.1]) and $\Omega_{/ R}$ (cusps) $=\Omega_{X_{\Gamma} / R}$ (cusps) the sheaf which, when restricted to the complement of the cuspidal sections, is the sheaf of regular differentials and whose sections in a neighborhood of the cuspidal sections are meromorphic differentials with at worst simple poles along those sections ([10, II 3]). We consider only rings $R$ which are flat over $\mathcal{O}$ or $\mathcal{O} / p^{n} \mathcal{O}$ for some $n$.

Proposition 1.1. ([24, Prop. 6.1], [6, Prop. 5.1]) Let $R \rightarrow R^{\prime}$ be a morphism of rings which are flat over $\mathcal{O}$ or $\mathcal{O} / p^{n} \mathcal{O}$ for some $n$. Then

$$
\begin{aligned}
H^{0}\left(X_{\Gamma / R}, \Omega_{/ R}\right) \otimes_{R} R^{\prime} & \cong H^{0}\left(X_{\Gamma / R^{\prime}}, \Omega_{/ R^{\prime}}\right), \\
H^{0}\left(X_{\Gamma / R}, \Omega_{/ R}(\text { cusps })\right) \otimes_{R} R^{\prime} & \cong H^{0}\left(X_{\Gamma / R^{\prime}}, \Omega_{/ R^{\prime}}(\text { cusps })\right) .
\end{aligned}
$$

We consider the $q$-expansions of regular differentials. Since $H^{0}\left(X_{\Gamma / R}, \Omega_{/ R}\right)=$ $H^{0}\left(X(N)_{/ R}, \Omega_{/ R}\right)^{H}$ by [5, VII 3.3], we can restrict ourselves to the case $\Gamma=\Gamma(N)$. Let $\operatorname{Tate}(q)$ be the Tate curve with $N$-sides over $\mathcal{O} \llbracket q^{\frac{1}{N}} \rrbracket$. Tate $(q)$ with Drinfeld basis $\left(\zeta_{N}, q^{\frac{1}{N}}\right)$ defines a point on $X_{\Gamma / \mathcal{O}}$, and the corresponding morphism

$$
\tau / \mathcal{O}: \operatorname{Spec} \mathcal{O} \llbracket q^{\frac{1}{N}} \rrbracket \rightarrow X_{\Gamma / \mathcal{O}}
$$

can be identified with the formal completion of $X_{\Gamma / \mathcal{O}}$ along the section corresponding to the cusp $\infty\left(=\left[\begin{array}{l}1 \\ 0\end{array}\right]\right)$ ([5, VII 2.4]). For any $R$ as above, we then have a morphism

$$
\tau_{/ R}: \operatorname{Spec} R \llbracket q^{\frac{1}{N}} \rrbracket \rightarrow X_{\Gamma / R} .
$$

For any $\omega \in H^{0}\left(X_{\Gamma / R}, \Omega_{/ R}\right)$, we define the $q$-expansion of $\omega$ at $\infty$ to be the element $\varphi_{R}(\omega) \in R \llbracket q^{\frac{1}{N}} \rrbracket$ such that

$$
\tau_{/ R}^{*} \omega=\varphi_{R}(\omega) d q^{\frac{1}{N}} / q^{\frac{1}{N}} .
$$

This defines the q-expansion morphism $\varphi_{R}: H^{0}\left(X_{\Gamma / R}, \Omega_{/ R}\right) \rightarrow R \llbracket q^{\frac{1}{N}} \rrbracket$. Similarly, there is a $q$-expansion map $\varphi_{R}: H^{0}\left(X_{\Gamma / R}, \Omega_{/ R}\right.$ (cusps) $) \rightarrow R \llbracket q^{\frac{1}{N}} \rrbracket$. Let $B^{0}(\mathcal{O})$ (resp. $B(\mathcal{O})$ ) be the submodule of $\mathcal{O} \llbracket q^{\frac{1}{N}} \rrbracket$ consisting of the $q$-expansions at $\infty$ of cusp forms (resp. holomorphic modular forms) of weight 2 on $\Gamma$ with coefficients in $\mathcal{O}$. For any $R$ as above, let $B^{0}(R)=B^{0}(\mathcal{O}) \otimes R$ and $B(R)=B(\mathcal{O}) \otimes R$. One can show, using Prop. 1.1, that $\varphi_{R}\left(H^{0}\left(X_{\Gamma / R}, \Omega_{/ R}\right)\right) \subset B^{0}(R)$ ([24, Prop. 6.2]). Similarly, we have $\varphi_{R}\left(H^{0}\left(X_{\Gamma / R}, \Omega_{/ R}(\right.\right.$ cusps $\left.\left.)\right)\right) \subset B(R)$. So we have maps

$$
\begin{array}{ccc}
\varphi_{R}: & H^{0}\left(X_{\Gamma / R}, \Omega_{/ R}\right) & \longrightarrow B^{0}(R), \\
\varphi_{R}: & H^{0}\left(X_{\Gamma / R}, \Omega_{/ R}(\text { cusps })\right) & \longrightarrow B(R) .
\end{array}
$$


One can define an action of $\mathbb{T}$ on $H^{0}\left(X_{\Gamma / R}, \Omega_{/ R}\right.$ (cusps) $)$ using the definition of Hecke correspondences, and one on $B(R)$ by its action on the $q$-expansions of classical modular forms. With these actions, (1.1) and (1.2) are then $\mathbb{T}$-morphisms. Furthermore, if $g \in R \llbracket q^{\frac{1}{N}} \rrbracket$ with zero constant term is a common eigenvector for $T_{l}(l \nmid N)$ and $U_{l}(l \mid N)$ with eigenvalues $c_{l}$, then the usual recursive relations ([18, (3.5.12)]) show that $g$ is determined by the $c_{l}$ up to multiplication by a constant.

1.3. $q$-expansions of $p$-torsion points. Let $X$ be a smooth projective curve defined over $\overline{\mathbb{F}}_{p}$ and let $J$ be its Jacobian. Let $\Omega_{X}^{1}$ be the canonical sheaf of differentials on $X$ and let $\mathcal{C}$ be the Cartier operator on $H^{0}\left(X, \Omega_{X}^{1}\right)([3])$. There is a canonical isomorphism (cf. [15, §11, Prop. 10])

$$
\delta: J[p] \rightarrow H^{0}\left(X, \Omega_{X}^{1}\right)^{\mathcal{C}}
$$

where $J[p]=\left\{x \in J\left(\overline{\mathbb{F}}_{p}\right): p x=0\right\}$ and $H^{0}\left(X, \Omega_{X}^{1}\right)^{\mathcal{C}}=\left\{\omega \in H^{0}\left(X, \Omega_{X}^{1}\right): \mathcal{C} \omega=\omega\right\}$. The definition of $\delta$ is as follows: if $x$ in the domain is represented by a divisor $D$ on $X_{/ \overline{\mathbb{F}}_{p}}$ such that $p D=(g)$ where $(g)$ is the divisor of $g$, then $\delta(x)=d g / g$.

Proposition 1.2. ([24, Prop. 6.5], [6, Prop. 5.2]) Let $J$ be the Jacobian of $X=$ $X_{\Gamma / \mathbb{F}_{p}}$ and let $\varphi_{\overline{\mathbb{F}}_{p}}$ be as in (1.1). Then $\varphi_{\overline{\mathbb{F}}_{p}} \circ(\delta \otimes 1)$ induces an injection $\varphi$ : $J[p] \otimes_{\mathbb{F}_{p}} \overline{\mathbb{F}}_{p} \hookrightarrow B^{0}\left(\overline{\mathbb{F}}_{p}\right)$ such that $\varphi \circ T_{n}^{*}=T_{n} \circ \varphi$ for all $n \geq 1$ where $T_{n}$ is the $n$-th Hecke operator and $T_{n}^{*}$ is the dual of the endomorphism of $J$ which $T_{n}$ induces by Pic functoriality.

We call $\varphi$ in Proposition 1.2 the $q$-expansion map of the $p$-torsion points of $J$. Let $A_{f}$ be the abelian subvariety over $\mathbb{Q}$ of the Jacobian $J_{\Gamma}$ of $X_{\Gamma}$ associated to $f\left(\left[18\right.\right.$, Thm. 7.14]). Let $K_{f}$ be the field generated over $\mathbb{Q}$ by the $a_{n}$ and $\iota$ the embedding $K_{f} \hookrightarrow \operatorname{End}\left(J_{\Gamma}\right) \otimes_{\mathbb{Z}} \mathbb{Q}$ from the construction of $A_{f}$ (loc. cit.). Let $\mathfrak{p}$ be the prime of $K_{f}$ below $\mathfrak{P}$.

Proposition 1.3. Assume that $\mathfrak{p} \nmid N$ and $f$ is ordinary at $\mathfrak{p}$. Then the image of $A_{f}[\mathfrak{p}]_{/ \mathbb{F}_{p}}\left(\overline{\mathbb{F}}_{p}\right) \otimes_{\mathbb{F}_{p}} \overline{\mathbb{F}}_{p}$ under $\varphi$ is the $\overline{\mathbb{F}}_{p}$-module generated by the reduction $\bar{f}$ of $f(q)$ in $B^{0}\left(\overline{\mathbb{F}}_{p}\right)$.

Proof. By [18, Thm. 7.14], $A_{f}$ is stable under subrings of $\operatorname{End} d_{\mathbb{Q}}\left(J_{\Gamma}\right)=\operatorname{End}\left(J_{\Gamma}\right) \otimes_{\mathbb{Z}} \mathbb{Q}$ induced by the Hecke correspondences $T_{n}$ via Albanese functoriality and Pic functoriality which are related as follows. The endomorphism of $J_{\Gamma}$ which $T_{n}$ induces by Albanese functoriality is the endomorphism denoted $\xi_{n}$ in [18, Chap. 7]. Its dual is the endomorphism of $J_{\Gamma}$ which $T_{n}$ induces by Pic functoriality. (For more details, see [14, p. 444].) By [18, Thm. 7.14(b)], $\xi_{n} x=\iota\left(a_{n}\right) x$ for all $x \in A_{f}$ and all $n$. Since $J_{\Gamma}$ has good reduction at $p$, the morphism $A_{f / \mathbb{Z}_{p}} \rightarrow J_{\Gamma / \mathbb{Z}_{p}}$ is a closed immersion ([11, Prop. 1.2]), hence $A_{f}[p]_{/ \mathbb{F}_{p}} \hookrightarrow J_{\Gamma}[p]_{/ \mathbb{F}_{p}}$. Since $f$ is ordinary at $\mathfrak{p}$, $A_{f}[\mathfrak{p}]_{/ \mathbb{F}_{p}}\left(\overline{\mathbb{F}}_{p}\right)$ is a one-dimensional vector space over the residue field of $\mathfrak{p}$ (cf. [25, Thm. 2.2]). The action of $\iota\left(a_{n}\right)$ on $A_{f}[\mathfrak{p}]_{/ \mathbb{F}_{p}}\left(\overline{\mathbb{F}}_{p}\right)$ is given by multiplication by $a_{n}$ $\bmod \mathfrak{p}$. For any $x$ in $A_{f}[\mathfrak{p}]_{/ \mathbb{F}_{p}}\left(\overline{\mathbb{F}}_{p}\right)$ and $n \geq 1$, it follows from Prop. 1.2 and the above discussion that

$$
T_{n} \varphi(x \otimes 1)=\varphi\left(T_{n}^{*} x \otimes 1\right)=\varphi\left(\iota\left(a_{n}\right) x \otimes 1\right)=a_{n} \varphi(x \otimes 1) .
$$

Now $\bar{f}$ is a common eigenvector for all $T_{n}$ with eigenvalues $a_{n} \bmod \mathfrak{p}$. Thus $\varphi(x \otimes 1), \bar{f} \in B^{0}\left(\overline{\mathbb{F}}_{p}\right)$ are two common eigenvectors of $T_{n}$ for all $n$ with the same eigenvalues. (Note that $B^{0}\left(\overline{\mathbb{F}}_{p}\right) \subset q \overline{\mathbb{F}}_{p} \llbracket q \rrbracket$.) So $\varphi(x \otimes 1)=c \bar{f}$ for some $c \in \overline{\mathbb{F}}_{p}^{*}$. This proves the proposition. 
Remark 1.4. Let $\sigma: K_{f} \hookrightarrow \mathbb{C}$ be an embedding. Let $f^{\sigma}$ be the cusp form obtained by applying $\sigma$ to the coefficients of $f$ (cf. [18, Thm. 7.14]), $A_{f}^{\sigma}$ the abelian subvariety of $J_{\Gamma}$ associated to $f^{\sigma}$ and $\mathfrak{p}^{\sigma}$ the $\sigma$-conjugate of $\mathfrak{p}$. Then $a_{p}^{\sigma}$ is a unit $\bmod \mathfrak{p}^{\sigma}$, so $f^{\sigma}$ is ordinary at $\mathfrak{p}^{\sigma}$. If $\sigma$ extends to an element in the decomposition group $D_{\mathfrak{P}}$ for $\mathfrak{P}$ (we view $K_{f}$ as a subfield of $\overline{\mathbb{Q}}$ ), then the same arguments as in Proposition 1.3 show that $\varphi\left(A_{f}^{\sigma}\left[\mathfrak{p}^{\sigma}\right]_{/ \mathbb{F}_{p}}\left(\overline{\mathbb{F}}_{p}\right) \otimes \overline{\mathbb{F}}_{p}\right)=\overline{\mathbb{F}}_{p} \cdot \overline{f^{\sigma}}$.

1.4. Cuspidal subgroups associated to Eisenstein series. We recall some results in [21] and [22] which we need below (Props. 1.5, 1.6 and 1.7). Let $\mathcal{E}_{\Gamma}$ be the space of weight 2 Eisenstein series on $\Gamma$. For any $E \in \mathcal{E}_{\Gamma}$, let $\omega_{E}$ be the differential form on $\mathfrak{H} / \Gamma$ whose pull-back to $\mathfrak{H}$ is $E(z) d z$. Let $\mathcal{P}(E)$ be the image of

$$
H_{1}(\mathfrak{H} / \Gamma, \mathbb{Z}) \rightarrow \mathbb{C}, \quad \gamma \mapsto \int_{\gamma} \omega_{E} .
$$

For any $\mathbb{Z}$-module $M \subset \mathbb{C}$, let $\mathcal{E}_{\Gamma}(M)=\left\{E \in \mathcal{E}_{\Gamma}: \mathcal{P}(E) \subset M\right\}$. We then have

Proposition 1.5. ([22, Prop. 1.1(a)]) For any $\mathbb{Z}$-module $M \subset \mathbb{C}$, the natural map $\mathcal{E}_{\Gamma}(\mathbb{Z}) \otimes_{\mathbb{Z}} M \rightarrow \mathcal{E}_{\Gamma}(M)$ is an isomorphism.

In $[21, \S 1.8]$, Stevens showed how one can associate to an arbitrary $E \in \mathcal{E}_{\Gamma}$ a subgroup $C_{E}$ of the cuspidal group $C_{\Gamma}$ of $J_{\Gamma}$. The construction of $C_{E}$ is as follows. Let cusps $=\operatorname{cusps}(\Gamma)$ denote the set of cusps on $X_{\Gamma}$ and $\operatorname{Div}^{0}$ (cusps) the group of divisors of degree zero supported on the cusps. For any $\mathbb{Z}$-module $M$, define $\operatorname{Div}^{0}($ cusps $; M)=\operatorname{Div}^{0}($ cusps $) \otimes_{\mathbb{Z}} M$. Let

$$
\delta_{\Gamma}(E)=\sum_{x \in \text { cusps }} r_{x}(E) \cdot x \quad \in \operatorname{Div}^{0}(\text { cusps } ; \mathbb{C})
$$

where $r_{x}(E)=2 \pi i \operatorname{res}_{x}\left(\omega_{E}\right)$ and $\operatorname{res}_{x}\left(\omega_{E}\right)$ is the residue of $\omega_{E}$ at the cusp $x$. We note that by $[22$, Thm $1.3(\mathrm{a})]$,

$$
r_{\left[\begin{array}{c}
r \\
s
\end{array}\right]}(E)=e\left(\left[\begin{array}{l}
r \\
s
\end{array}\right]\right) \cdot a_{0}\left(E \mid \gamma_{\left[\begin{array}{c}
r \\
s
\end{array}\right]}\right)
$$

where $e\left(\left[\begin{array}{l}r \\ s\end{array}\right]\right)$ is the ramification index of $\left[\begin{array}{l}r \\ s\end{array}\right]$ over $X(1), \gamma_{\left[\begin{array}{l}r \\ s\end{array}\right]} \in \mathrm{SL}_{2}(\mathbb{Z})$ is such that $\gamma_{\left[\begin{array}{l}r \\ s\end{array}\right]} \cdot i \infty$ represents $\left[\begin{array}{l}r \\ s\end{array}\right]$ and $a_{0}\left(E \mid \gamma_{\left[\begin{array}{c}r \\ s\end{array}\right]}\right)$ is the constant term of the Fourier expansion of $E \mid \gamma_{\left[{ }_{s}^{r}\right]}$. Let $\mathcal{R}(E)$ be the $\mathbb{Z}$-submodule of $\mathbb{C}$ generated by the coefficients of $\delta_{\Gamma}(E)$ and let

$$
\mathcal{R}(E)^{*}=\left\{\eta \in \operatorname{Hom}_{\mathbb{Q}}\left(\mathcal{R}(E) \otimes_{\mathbb{Z}} \mathbb{Q}, \mathbb{Q}\right): \eta(\mathcal{R}(E)) \subset \mathbb{Z}\right\} .
$$

The subgroup $C_{E}$ of $C_{\Gamma}$ associated to $E$ is by definition the image of the composition

$$
\begin{aligned}
& \mathcal{R}(E)^{*} \longrightarrow \operatorname{Div}^{0}(\text { cusps }) \stackrel{\theta}{\longrightarrow} C_{\Gamma}, \\
& \eta \quad \longmapsto \quad \eta\left(\delta_{\Gamma}(E)\right) \text {, }
\end{aligned}
$$

where $\theta$ sends a divisor to its divisor class. Note that since $r_{x}(E)$ is the integral of $\omega_{E}$ along some cycle around $x, \mathcal{R}(E) \subset \mathcal{P}(E)$. Let $A_{E}=\mathcal{P}(E) / \mathcal{R}(E)$.

Proposition 1.6. ([22, Thm. 1.2(a)]) For any $E \in \mathcal{E}_{\Gamma}$, there is a perfect duality $C_{E} \times A_{E} \rightarrow \mathbb{Q} / \mathbb{Z}$.

Suppose $E \in \mathcal{E}_{\Gamma}$ is a common eigenfunction for all $T_{l}$ and $\langle l\rangle, l \nmid N$. Then there are Dirichlet characters $\epsilon_{1}$ and $\epsilon_{2}$ modulo $N$ such that $E \mid T_{l}=\left(\epsilon_{1}(l)+l \epsilon_{2}(l)\right) E$ for each prime $l \nmid N([21,3.2 .2,(3.2 .3)])$. We say that $E$ has signature $\epsilon_{1}, \epsilon_{2}$. Let $\mathbb{Z}\left[\epsilon_{1}, \epsilon_{2}\right]$ (resp. $\mathbb{Q}\left[\epsilon_{1}, \epsilon_{2}\right]$ ) be the ring generated by the values of $\epsilon_{1}$ and $\epsilon_{2}$ over $\mathbb{Z}$ (resp. $\mathbb{Q}$ ). By [21, 3.2.1, 3.2.2], $\mathcal{P}(E)$ and $\mathcal{R}(E)$ are fractional ideals of 
$\mathbb{Q}\left[\epsilon_{1}, \epsilon_{2}\right]$. Let $\mathfrak{o}=\mathbb{Z}\left[1 / 2, \epsilon_{1}, \epsilon_{2}\right], \mathfrak{a}=\mathfrak{o}+\mathfrak{o} \mathcal{R}(E)$ and $\mathfrak{b}$ the $\mathfrak{o}$-module generated by $\left\{1, B_{1, \epsilon_{1}^{-1}}, B_{1, \epsilon_{2}}, S\left(\epsilon_{1}\right) B_{2, \epsilon_{2}}, S\left(\epsilon_{2}\right) B_{2, \epsilon_{1}^{-1}}\right\}$, where for $i=1,2, B_{i,-}$ are the generalized Bernoulli numbers and

$$
S\left(\epsilon_{i}\right)= \begin{cases}\phi\left(N_{i}\right) & \text { if } \epsilon_{i}=1 \\ 0 & \text { otherwise }\end{cases}
$$

and $\phi$ is the Euler function.

Proposition 1.7. ([21, Thm. 3.6.1]) Let $E \in \mathcal{E}_{\Gamma}$ have signature $\epsilon_{1}, \epsilon_{2}$. Then $\mathfrak{a} \subset \mathfrak{o} \mathcal{P}(E) \subset \mathfrak{b}$.

We next give a set of generators of the space over $\mathbb{Q}$ of weight 2 Eisenstein series $E$ of level $N$ with $\mathcal{R}(E) \subset \mathbb{Q}$. For fractional ideals $\mathfrak{a}_{1}$ and $\mathfrak{a}_{2}$ of $\mathbb{Q}$ and $a_{1}, a_{2} \in \mathbb{Q}$, define

$$
\begin{aligned}
E(z, s) & =E\left(z, s ; a_{1}, a_{2} ; \mathfrak{a}_{1}, \mathfrak{a}_{2}\right) \\
& =-N\left(\mathfrak{a}_{2}\right)(2 \pi)^{-2} \sum_{\left(m_{1}, m_{2}\right)}\left(m_{1} z+m_{2}\right)^{-2}\left|m_{1} z+m_{2}\right|^{-2 s}
\end{aligned}
$$

for $z \in \mathfrak{H}, s \in \mathbb{C}$ with $\operatorname{Re} s>2$, where the sum is over all pairs $\left(m_{1}, m_{2}\right) \in \mathbb{Q}^{2}-(0,0)$ such that $m_{i} \equiv a_{i}\left(\bmod \mathfrak{a}_{i}\right), i=1,2$. For fixed $z, E(z, s)$ may be continued analytically to a meromorphic function in the $s$-plane which is holomorphic at $s=0$ (cf. $[19, \S 3])$. Define

$$
E(z)=E\left(z ; a_{1}, a_{2} ; \mathfrak{a}_{1}, \mathfrak{a}_{2}\right)=E\left(z, 0 ; a_{1}, a_{2} ; \mathfrak{a}_{1}, \mathfrak{a}_{2}\right) .
$$

The Fourier expansion of $E(z)$ at $\infty$ is given by

$$
-\delta\left(a_{1}, \mathfrak{a}_{1}\right)(2 \pi)^{-2} N\left(\mathfrak{a}_{2}\right) \sum_{0 \neq d \equiv a_{2}}|d|^{-2}+\sum_{c \equiv a_{1}} \sum_{\substack{\left(\bmod \mathfrak{a}_{1}\right) \\ b c>0}} \sum_{b \in \mathfrak{a}_{2}-1}|b| e^{2 \pi i\left(b c z+b a_{2}\right)}
$$

where $\delta\left(a_{1}, \mathfrak{a}_{1}\right)=1$ or 0 according as $a_{1} \in \mathfrak{a}_{1}$ or not (cf. [19, (3.6)]). For any $(x, y) \in\left(N^{-1} \mathbb{Z} / \mathbb{Z}\right)^{2}$, let

$$
\phi_{(x, y)}(z)=N^{-2} \sum_{\left(a_{1}, a_{2}\right) \in\left(N^{-1} \mathbb{Z} / \mathbb{Z}\right)^{2}} e^{2 \pi i\left(a_{2} x_{1}-a_{1} x_{2}\right)} E\left(z ; a_{1}, a_{2} ; \mathbb{Z}, \mathbb{Z}\right) .
$$

By Hecke (cf. [21, pp. 59-60]), $\left\{\phi_{(x, y)}:(x, y) \in\left(N^{-1} \mathbb{Z} / \mathbb{Z}\right)^{2}-(0,0)\right\}$ spans the space of weight 2 Eisenstein series $E$ of level $N$ with $\mathcal{R}(E) \subset \mathbb{Q}$ over $\mathbb{Q}$. Another fact we need is that if $U_{\Gamma}$ is the group of meromorphic functions on $X_{\Gamma / \mathbb{C}}$ whose divisors are supported on the cusps, then logarithmic differentiation gives an isomorphism

$$
U_{\Gamma} / \mathbb{C}^{*} \stackrel{\sim}{\longrightarrow} \mathcal{E}_{\Gamma}(\mathbb{Z}), \quad g \mapsto \frac{1}{2 \pi i} \frac{g^{\prime}(z)}{g(z)},
$$

and if $E(z)=(2 \pi i)^{-1} g^{\prime}(z) / g(z)$, then $\delta_{\Gamma}(E)=(g)$ (cf. [22, §1]).

Lemma 1.8. For any $E \in \mathcal{E}_{\Gamma}(\mathbb{Z})$, the Fourier coefficients of $E$ at each cusp are in $\left(12 N^{2}\right)^{-1} \mathbb{Z}$.

Proof. By (1.9) and a theorem of Kubert (cf. [22, §3]), $2 E$ is a $\mathbb{Z}$-linear combination of the $\phi_{(x, y)}$ with $(x, y) \in\left(N^{-1} \mathbb{Z} / \mathbb{Z}\right)^{2}-(0,0)$. For any $\gamma=\left(\begin{array}{ll}a & b \\ c & d\end{array}\right) \in \mathrm{SL}_{2}(\mathbb{Z})$, we have $\phi_{(x, y)} \mid \gamma=\phi_{(x, y) \mid \gamma}$, where $(x, y) \mid \gamma=(a x+c y, b x+d y)$ (cf. [21, 2.4.1(a)]). From $[21,2.4 .2(\mathrm{a})]$, we see that the Fourier coefficients of $\phi_{(x, y)},(x, y) \in\left(N^{-1} \mathbb{Z} / \mathbb{Z}\right)^{2}-$ $(0,0)$, at $\infty$ are in $\left(12 N^{2}\right)^{-1} \mathbb{Z}$. 
Suppose now $E$ is a common eigenfunction for $T_{l}$ for all $l$ and $\langle l\rangle, l \nmid N$, such that $\mathfrak{P}$ is an Eisenstein prime associated to $f$ and $E$ as at the beginning of $\S 1$.

Proposition 1.9. Assume that $\mathfrak{p} \nmid 6 N$ and $f$ is ordinary at $\mathfrak{p}$. Then $p$ divides the order of $C_{E}$.

Proof. Let $\mathfrak{o}_{1}=\mathbb{Z}\left[\epsilon_{0}^{\prime}, \epsilon_{0} \chi^{-1}\right]$ and $\mathfrak{o}=\mathfrak{o}_{1}[1 / 2]$. By Prop. 1.7, $N \cdot \mathcal{P}(E) \subset \mathfrak{o}$, so $E \in \mathcal{E}_{\Gamma}\left(N^{-1} \mathfrak{o}\right)$. By Prop. 1.5, there exist $\lambda_{i} \in N^{-1} \mathfrak{o}, E_{i} \in \mathcal{E}_{\Gamma}(\mathbb{Z})$ such that $E=\sum_{i} \lambda_{i} E_{i}$. Since $\mathfrak{b} \subset N^{-1} \mathfrak{o}$, Prop. 1.7 shows that the non-integral part of $\mathfrak{o} \mathcal{P}(E)$ is prime to $p$. (By the integral (resp. non-integral) part of a fractional ideal, we mean the product of the factors in its prime decomposition which occur to a positive (resp. negative) exponent.) Since $\mathfrak{o} \mathcal{R}(E) \subset \mathfrak{o} \mathcal{P}(E)$, the non-integral part of $\mathfrak{o} \mathcal{R}(E)$ is prime to $p$ also. We suppose $p \nmid \# C_{E}$ and derive a contradiction. By Prop. 1.6, $p \nmid \#(\mathcal{P}(E) / \mathcal{R}(E))$, so $p \nmid \#(\mathfrak{o} \mathcal{P}(E) / \mathfrak{o} \mathcal{R}(E))$. Since $\mathfrak{a} \subset \mathfrak{o} \mathcal{P}(E)$ by Prop. $1.7, p \nmid \#(\mathfrak{o}+\mathfrak{o} \mathcal{R}(E)) / \mathfrak{o} \mathcal{R}(E)=\# \mathfrak{o} /(\mathfrak{o} \cap \mathfrak{o} \mathcal{R}(E))$. This implies that the integral part of $\mathfrak{o} \mathcal{R}(E)$ is prime to $p$. Hence $\mathfrak{o} \mathcal{R}(E)$ is prime to $p$.

Let $\wp=\mathfrak{P} \cap \mathfrak{o}_{1}$ and let $\bar{\eta}_{0}$ be the composite homomorphism $\mathfrak{o}_{1} \rightarrow \mathfrak{o}_{1} / \wp \stackrel{\operatorname{tr}}{\rightarrow} \mathbb{F}_{p}$ where tr is the trace map from $\mathbb{F}:=\mathfrak{o}_{1} / \wp$ to $\mathbb{F}_{p}$. Since $\mathfrak{o}_{1}$ is projective over $\mathbb{Z}$, we can lift $\bar{\eta}_{0}$ to a surjective homomorphism $\eta_{0}: \mathfrak{o}_{1} \rightarrow \mathbb{Z}$. Extend $\eta_{0} \mathbb{Q}$-linearly to a $\operatorname{map} \mathcal{R}(E) \otimes \mathbb{Q} \rightarrow \mathbb{Q}$, denoted $\eta_{0}$ again. Since $\mathcal{R}(E)$ is prime to $p$, there exists $a \in \mathbb{Z}$, $(a, p)=1$, such that $a \mathcal{R}(E) \subset \mathfrak{o}_{1}$. Since $a \mathcal{R}(E)$ is prime to $p, \eta_{0}(a \mathcal{R}(E))=m \mathbb{Z}$ for some $m \in \mathbb{Z}$ prime to $p$. Let $\eta=\frac{a}{m} \eta_{0}$. Then $\eta \in \mathcal{R}(E)^{*}$ satisfies $\eta(\mathcal{R}(E))=\mathbb{Z}$, $\eta(\mathfrak{o})=\frac{a}{m} \mathbb{Z}[1 / 2]$ and $\eta(\wp) \subset \frac{p}{m} \mathbb{Z}$. So $\eta\left(N^{-1} \mathfrak{o}\right)=\frac{a}{m N} \mathbb{Z}[1 / 2]$, and we can choose $n \in \mathbb{Z}, p \nmid n$, such that $n_{i}=n \eta\left(\lambda_{i}\right) \in \mathbb{Z}$ for all $i$. Let

$$
\eta(E)=\sum_{i} \eta\left(\lambda_{i}\right) E_{i}
$$

Then

$$
\eta(E)(q)=\sum_{i} \eta\left(\lambda_{i}\right) E_{i}(q)=\eta\left(\sum_{i} \lambda_{i} E_{i}(q)\right)=\eta(E(q)),
$$

where $\eta(E(q))$ is the $q$-series obtained by applying $\eta$ to the coefficients of $E(q)$. Since $f \equiv E(\bmod \mathfrak{P})$,

$$
\eta(E)(q) \equiv \operatorname{Tr}_{\mathbb{F} / \mathbb{F}_{p}}(E(q)) \equiv \operatorname{Tr}_{\mathbb{F} / \mathbb{F}_{p}}(f(q))\left(\bmod p \mathbb{Z}_{(p)}\right),
$$

where $\operatorname{Tr}_{\mathbb{F} / \mathbb{F}_{p}}(E(q))$ is the $q$-series obtained by reducing the coefficients of $E(q) \bmod$ $\wp$ and taking the trace from $\mathbb{F}$ to $\mathbb{F}_{p}$, and similarly for $\operatorname{Tr}_{\mathbb{F} / \mathbb{F}_{p}}(f(q))$, and $\mathbb{Z}_{(p)}$ is the localization of $\mathbb{Z}$ at $p$. By (1.9), there exist $g_{i} \in U_{\Gamma}$ such that $(2 \pi i)^{-1} g_{i}^{\prime}(z) / g_{i}(z)=$ $E_{i}(z)$ for all $i$. Let $g=\prod_{i} g_{i}^{n_{i}}$ and $\omega=d g / g$. Then $n \eta(E)(q)$ is the $q$-expansion of $\omega$ at $\infty$ and $(g)=\delta_{\Gamma}(n \eta(E))$. In particular, $g$ is non-constant. Since $\eta(E)=$ $\sum_{i} \eta\left(\lambda_{i}\right) E_{i}$ with $E_{i} \in \mathcal{E}_{\Gamma}(\mathbb{Z})$ and $\eta\left(\lambda_{i}\right) \in \mathbb{Z}_{(p)}$ for all $i$, Lemma 1.8 gives that $\eta(E)$ has $p$-integral Fourier coefficients at each cusp for $p \nmid 6 N$. It follows from [5, VII 3.9(ii)] that $\eta(E)$ is a modular form with coefficients in $\mathcal{O}$. (Recall $\mathcal{O}$ was the completion of $\mathbb{Z}\left[\zeta_{N}\right]$ at the prime below $\mathfrak{P}$.) Hence $\omega$ arises by extension of scalars to $\mathbb{C}$ from an element (denoted $\omega$ again) in $H^{0}\left(X_{1}(N) / \mathcal{O}, \Omega_{/ \mathcal{O}}(\right.$ cusps)) with $\varphi_{\mathcal{O}}(\omega)=n \eta(E)(q)$.

Write $X$ for $X_{\Gamma}$. Let $\mathcal{O}^{*}$ (cusps) denote the sheaf on $X_{/ \mathcal{O}}$ which when restricted to the complement of the cuspidal divisors is the sheaf of invertible elements of $\mathcal{O}_{X}$ and whose sections in a neighborhood of the cuspidal divisors consist of functions with divisors supported on those divisors. We see from the exact sequence

$$
0 \rightarrow \mathcal{O}^{*} \rightarrow H^{0}\left(X_{/ \mathcal{O}}, \mathcal{O}^{*}(\text { cusps })\right) \stackrel{d \log }{\longrightarrow} H^{0}\left(X_{/ \mathcal{O}}, \Omega_{/ \mathcal{O}}(\text { cusps })\right)
$$


that $g$ comes from a function (denoted $g$ again) in $H^{0}\left(X_{/ \mathcal{O}}, \mathcal{O}^{*}\right.$ (cusps)) up to an element in $\mathcal{O}^{*}$. Let $g_{0}$ be the function on $X_{/ \overline{\mathbb{F}}_{p}}$ obtained from $g$ by the base change $\operatorname{Spec}\left(\overline{\mathbb{F}}_{p}\right) \rightarrow \operatorname{Spec}(\mathcal{O})$. Then $g_{0}$ is a non-constant function. Since $X_{/ \mathcal{O}}$ is smooth over $\operatorname{Spec}(\mathcal{O}),\left(g_{0}\right)=\delta_{\Gamma}(n \eta(E))_{/ \overline{\mathbb{F}}_{p}}$ by $\left[17\right.$, Thm. 20], where $\delta_{\Gamma}(n \eta(E))_{/ \overline{\mathbb{F}}_{p}}$ is the pull-back of $\delta_{\Gamma}(n \eta(E))$ to $X_{/ \overline{\mathbb{F}}_{p}}$. (The cuspidal sections of $X_{/ \mathbb{Z}\left[1 / N, \zeta_{N}\right]}$ are all disjoint over Spec $\mathbb{Z}\left[1 / N, \zeta_{N}\right]$, cf. $[5$, VII $\S 2]$.) Let $\omega_{/ \overline{\mathbb{F}}_{p}}$ be the image of $\omega$ in $H^{0}\left(X_{/ \overline{\mathbb{F}}_{p}}, \Omega_{/ \overline{\mathbb{F}}_{p}}(\right.$ cusps $\left.)\right)$. By $(1.10), \varphi_{\overline{\mathbb{F}}_{p}}\left(\omega_{/ \overline{\mathbb{F}}_{p}}\right)=n \cdot \operatorname{Tr}_{\mathbb{F} / \mathbb{F}_{p}}(f(q))$. For each $\tau \in \operatorname{Gal}\left(\mathbb{F} / \mathbb{F}_{p}\right)$, fix a lift $\sigma_{\tau}$ of $\tau$ in the decomposition group $D_{\mathfrak{P}}$ of $G_{\mathbb{Q}}$ for $\mathfrak{P}$, and let $x_{\tau} \otimes c_{\tau} \in A_{f}^{\sigma_{\tau}}\left[\mathfrak{p}^{\sigma_{\tau}}\right]_{/ \mathbb{F}_{p}}\left(\overline{\mathbb{F}}_{p}\right) \otimes \overline{\mathbb{F}}_{p}$ be such that $\varphi\left(x_{\tau} \otimes c_{\tau}\right)=\overline{f^{\sigma_{\tau}}}$ (cf. Prop. 1.3, Remark 1.4). Then

$$
\varphi_{\overline{\mathbb{F}}_{p}}\left(\omega_{/ \overline{\mathbb{F}}_{p}}\right)=n \sum_{\tau} \overline{f^{\sigma_{\tau}}}=n \cdot \varphi\left(\sum_{\tau} x_{\tau} \otimes c_{\tau}\right) \in \varphi\left(J_{\Gamma}[p]_{/ \mathbb{F}_{p}}\left(\overline{\mathbb{F}}_{p}\right) \otimes \overline{\mathbb{F}}_{p}\right)
$$

and so $\omega_{/ \mathbb{F}_{p}} \in H^{0}\left(X_{/ \mathbb{F}_{p}}, \Omega_{X}^{1}\right)^{\mathcal{C}} \otimes \overline{\mathbb{F}}_{p}$. Since $\omega_{/ \overline{\mathbb{F}}_{p}}$ is of the form $d g_{0} / g_{0}, \omega_{/ \overline{\mathbb{F}}_{p}} \in$ $H^{0}\left(X_{/ \overline{\mathbb{F}}_{p}}, \Omega_{X}^{1}\right)$ by $\left[3\right.$, Thm. 2]. Hence there exists $x \in A_{f}[p]_{/ \mathbb{F}_{p}}\left(\overline{\mathbb{F}}_{p}\right)$ such that $\delta(x)=\omega_{/ \overline{\mathbb{F}}_{p}}$ by (1.3). If $x$ is represented by a divisor $D$ on $X_{/ \overline{\mathbb{F}}_{p}}$, then $p n D \equiv\left(g_{0}\right)$ $\left(\bmod p \operatorname{Div}^{0}\left(X_{/ \overline{\mathbb{F}}_{p}}\right)\right)$, where $\operatorname{Div}^{0}\left(X_{/ \overline{\mathbb{F}}_{p}}\right)$ is the group of divisors of degree 0 on $X_{/ \overline{\mathbb{F}}_{p}}$. Since $p \nmid n$ and $\delta_{\Gamma}(n \eta(E))=n \delta_{\Gamma}(\eta(E)), p D \equiv \delta_{\Gamma}(\eta(E))_{/ \overline{\mathbb{F}}_{p}}\left(\bmod p \operatorname{Div}^{0}\left(X_{/ \overline{\mathbb{F}}_{p}}\right)\right)$. Hence the coefficients of $\delta_{\Gamma}(\eta(E))_{/ \overline{\mathbb{F}}_{p}}$ are all divisible by $p$. But this contradicts the fact that $\eta(\mathcal{R}(E))=\mathbb{Z}$. Hence $p \mid \# C_{E}$. This proves Proposition 1.9.

Let $J_{\Gamma / \mathbb{Z}}$ be the Néron model of $J_{\Gamma / \mathbb{Q}}$. Let $C_{\Gamma / \mathbb{Z}}\left(\right.$ resp. $\left.C_{E / \mathbb{Z}}\right)$ be the schemetheoretic closure of $C_{\Gamma}$ (resp. $\left.C_{E}\right)$ in $J_{\Gamma / \mathbb{Z}}$.

Corollary 1.10. With notation as above, $C_{E}[p]_{/ \mathbb{F}_{p}}\left(\overline{\mathbb{F}}_{p}\right) \neq 0$.

Proof. Since the cusps of $X_{1}(N)$ are rational over $\mathbb{Q}\left(\zeta_{N}\right)$ and $p \nmid N, C_{E}$ is unramified at $p$. Thus $C_{E / \mathbb{Z}_{p}}$ is a Néron model of $C_{E / \mathbb{Q}_{p}}$ ([1, 7.1, Cor. 6]). By Prop. 1.9, there exists $x \in C_{E / \mathbb{Z}_{p}}(K)$ of exact order $p$, where $K$ is the field of fractions of $\mathcal{O}$. Let $\tilde{x} \in C_{E / \mathbb{Z}_{p}}(\mathcal{O})$ be the $\mathcal{O}$-valued point corresponding to $x$. Since $C_{E}[p]_{/ \mathbb{Z}_{p}}$ is finite flat, the specialization lemma in $[11, \S 1]$ shows that the specialization of $\tilde{x}$ to the special fiber has order $p$, so $C_{E}[p]_{/ \mathbb{F}_{p}}\left(\overline{\mathbb{F}}_{p}\right) \neq 0$.

Next we determine the image of $C_{E}[p]_{/ \mathbb{F}_{p}}\left(\overline{\mathbb{F}}_{p}\right)$ under $\varphi$.

Lemma 1.11. For any prime $l$ and any $E \in \mathcal{E}_{\Gamma}$, we have

$$
T_{l}^{*}\left(\delta_{\Gamma}(E)\right)=\delta_{\Gamma}\left(E \mid T_{l}\right)
$$

where $T_{l}^{*}$ acts on $\delta_{\Gamma}(E)$ via its action on the cusps.

Proof. Let $\pi: X(N) \rightarrow X_{\Gamma}$ be the natural projection. The induced map

$$
\pi^{*}: \operatorname{Div}^{0}(\operatorname{cusps}(\Gamma)) \longrightarrow \operatorname{Div}^{0}(\operatorname{cusps}(\Gamma(N)))
$$

is injective. It is easy to check that $\pi^{*}$ commutes with the actions of $T_{l}^{*}$. Thus it is enough to prove the lemma with $\Gamma=\Gamma(N)$. As $\left\{\phi_{(x, y)}:(x, y) \in\left(N^{-1} \mathbb{Z} / \mathbb{Z}\right)^{2}-\right.$ $(0,0)\}$ spans the space of weight 2 Eisenstein series $E$ of level $N$ with $\mathcal{R}(E) \subset \mathbb{Q}$ over $\mathbb{Q}$, it suffices by Prop. 1.5 to prove the lemma for $E=\phi_{(x, y)},(x, y) \in$ $\left(N^{-1} \mathbb{Z} / \mathbb{Z}\right)^{2}-(0,0)$, and all primes $l$. For $l \nmid N$, the lemma follows from [21, 1.3.2, 2.4.7, 3.2.1]. 
Suppose now $l \mid N$. We have the double coset decomposition:

$$
\Gamma\left(\begin{array}{ll}
l & 0 \\
0 & 1
\end{array}\right) \Gamma=\bigcup_{k=0}^{l-1}\left(\begin{array}{cc}
l & 0 \\
N k & 1
\end{array}\right) \Gamma .
$$

So

$$
\begin{aligned}
T_{l}^{*} \delta_{\Gamma}\left(\phi_{(x, y)}\right) & =\sum_{\left[\begin{array}{c}
r \\
s
\end{array}\right] \in \mathbf{c u s p s}} r_{\left[\begin{array}{l}
r \\
s
\end{array}\right]}\left(\phi_{(x, y)}\right) \cdot \sum_{k=0}^{l-1}\left[\begin{array}{c}
l r \\
N k r+s
\end{array}\right] \\
& =\sum_{\left[\begin{array}{c}
r \\
s
\end{array}\right]}\left(\sum r_{\left[\begin{array}{l}
\left.r^{\prime}\right] \\
s^{\prime}
\end{array}\right]}\left(\phi_{(x, y)}\right)\right)\left[\begin{array}{l}
r \\
s
\end{array}\right] \\
& =\sum_{\left[\begin{array}{l}
r \\
s
\end{array}\right]} \sum_{k=0}^{l-1} r_{\left[\begin{array}{l}
r+N k s \\
l s
\end{array}\right]}\left(\phi_{(x, y)}\right)\left[\begin{array}{l}
r \\
s
\end{array}\right],
\end{aligned}
$$

where the unindexed sum is over all $\left[\begin{array}{c}r^{\prime} \\ s^{\prime}\end{array}\right] \in \operatorname{cusps}=\operatorname{cusps}(\Gamma(N))$ such that $\left[\begin{array}{c}l r^{\prime} \\ N k r^{\prime}+s^{\prime}\end{array}\right]=\left[\begin{array}{l}r \\ s\end{array}\right]$ for some $0 \leq k \leq l-1$. Let $S=\left\{\left[\begin{array}{l}r \\ s\end{array}\right] \in\right.$ cusps : $\left.l \nmid r\right\}$ and $S^{\prime}=$ cusps $-S$. We split the sum over $\left[\begin{array}{l}r \\ s\end{array}\right]$ in (1.11) into two sums $\Sigma_{1}$ and $\Sigma_{2}$ over $S$ and $S^{\prime}$ respectively. By [21, Props. 2.4.1(a), 2.4.2(a)], $a_{0}\left(\phi_{(x, y)} \mid \gamma_{\left[\begin{array}{l}r \\ s\end{array}\right]}\right)=$ $\frac{1}{2} B_{2}(r x+s y)$, where $B_{2}(t)$ is the second Bernoulli function. We remark that $B_{2}(t)$ is periodic with period 1 . So from (1.4),

$$
r_{\left[\begin{array}{c}
r \\
s
\end{array}\right]}\left(\phi_{(x, y)}\right)=\frac{1}{2} B_{2}(r x+s y) \text {. }
$$

For $\left[\begin{array}{l}r \\ s\end{array}\right] \in S$ we have $\left[\begin{array}{c}r+N k s \\ l s\end{array}\right]=\left[\begin{array}{l}r \\ l s\end{array}\right]$, so

$$
\begin{aligned}
r_{\left[\begin{array}{l}
r+N k s \\
l s
\end{array}\right]}\left(\phi_{(x, y)}\right)=r_{\left[\begin{array}{l}
r \\
l s
\end{array}\right]}\left(\phi_{(x, y)}\right) & =\frac{1}{2} e\left(\left[\begin{array}{l}
r \\
l s
\end{array}\right]\right) B_{2}(r x+l s y)=\frac{1}{l} e\left(\left[\begin{array}{l}
r \\
s
\end{array}\right]\right) a_{0}\left(\phi_{(x, l y)} \mid \gamma_{\left[\begin{array}{l}
r \\
s
\end{array}\right]}\right) \\
& =\frac{1}{l} r_{\left[\begin{array}{l}
r \\
s
\end{array}\right]}\left(\phi_{(x, l y)}\right)
\end{aligned}
$$

since $e\left(\left[\begin{array}{c}r \\ l s\end{array}\right]\right)=e\left(\left[\begin{array}{c}r \\ s\end{array}\right]\right) / l$ for $l \mid N$ and since $l \nmid r$. Hence

$$
\Sigma_{1}=\sum_{\left[\begin{array}{l}
r \\
s
\end{array}\right] \in S} r_{\left[\begin{array}{l}
r \\
s
\end{array}\right]}\left(\phi_{(x, l y)}\right)\left[\begin{array}{l}
r \\
s
\end{array}\right] .
$$

For $\left[\begin{array}{l}r \\ s\end{array}\right] \in S^{\prime}$ we have $\left[\begin{array}{c}r+N k s \\ l s\end{array}\right]=\left[\begin{array}{c}r / l+N k s / l \\ s\end{array}\right]$, so by $(1.12)$

$$
\Sigma_{2}=l \sum_{\left[\begin{array}{l}
r \\
s
\end{array} \in S^{\prime}\right.} \sum_{k=0}^{l-1} e\left(\left[\begin{array}{l}
r \\
s
\end{array}\right]\right) \frac{1}{2} B_{2}(r x / l+N x k s / l+s y) .
$$

Write $x=a / N_{2}$ with $N_{2} \mid N$ and $\left(a, N_{2}\right)=1$. We now divide into two subcases according as $l$ divides $N / N_{2}$ or not. Suppose first $l \nmid\left(N / N_{2}\right)$. Then $l \nmid a$, so

$$
\Sigma_{2}=l \sum_{\left[\begin{array}{l}
r \\
s
\end{array}\right] \in S^{\prime}} \sum_{k=0}^{l-1} e\left(\left[\begin{array}{l}
r \\
s
\end{array}\right]\right) \frac{1}{2} B_{2}(r x / l+k s / l+s y)\left[\begin{array}{l}
r \\
s
\end{array}\right] .
$$

By [21, Prop. 2.4.7] and its proof, we have

$$
\phi_{(x, y)} \mid T_{l}=\phi_{(x, l y)}=\sum_{k=0}^{l-1} \sum_{j=0}^{l-1} \phi_{((x+j) / l, y+k / l)} .
$$


For $\left[\begin{array}{l}r \\ s\end{array}\right] \in S^{\prime}, 0 \leq k \leq l-1$ and $0 \leq j \leq l-1$, we have

$$
\begin{aligned}
r_{\left[\begin{array}{l}
r \\
s
\end{array}\right]}\left(\phi_{((x+j) / l, y+k / l)}\right) & =e\left(\left[\begin{array}{l}
r \\
s
\end{array}\right]\right) \frac{1}{2} B_{2}\left(\frac{x+j}{l} r+\left(y+\frac{k}{l}\right) s\right) \\
& =e\left(\left[\begin{array}{l}
r \\
s
\end{array}\right]\right) \frac{1}{2} B_{2}\left(\frac{x r}{l}+y s+\frac{k s}{l}\right) .
\end{aligned}
$$

Putting (1.11), (1.13), (1.15)-(1.17) together, we have $T_{l}^{*} \delta_{\Gamma}\left(\phi_{(x, y)}\right)=\delta_{\Gamma}\left(\phi_{(x, y)} \mid T_{l}\right)$ for $l \nmid\left(N / N_{2}\right)$.

Suppose now $l \mid\left(N / N_{2}\right)$. The proof of [21, Prop. 2.4.7] shows that

$$
\phi_{(x, y)} \mid T_{l}=l \sum_{k=0}^{l-1} \phi_{((x+k) / l, y)} .
$$

By (1.13), [21, Prop. 2.4.2(b)] and (1.12), we have

$$
\begin{aligned}
\Sigma_{1} & =\sum_{\left[\begin{array}{l}
r \\
s
\end{array}\right] \in S} \sum_{k=0}^{l-1} \sum_{j=0}^{l-1} r_{\left[\begin{array}{c}
r \\
s
\end{array}\right]}(\phi((x+k) / l, y+j / l))\left[\begin{array}{l}
r \\
s
\end{array}\right] \\
& =\sum_{\left[\begin{array}{l}
r \\
s
\end{array}\right] \in S} e\left(\left[\begin{array}{l}
r \\
s
\end{array}\right]\right) \sum_{k=0}^{l-1} \sum_{j=0}^{l-1} \frac{1}{2} B_{2}(r x / l+s y+k r / l+s j / l) .
\end{aligned}
$$

As $k$ and $j$ run through $\{0,1, \cdots, l-1\}, k r+s j(\bmod l)$ runs through $\{0,1, \cdots, l-1\}$ $l$ times. Thus (1.19) and (1.12) give

$$
\begin{aligned}
\Sigma_{1} & =l \sum_{\left[\begin{array}{l}
r \\
s
\end{array}\right] \in S} e\left(\left[\begin{array}{l}
r \\
s
\end{array}\right]\right) \sum_{k=0}^{l-1} \frac{1}{2} B_{2}(r x / l+k / l+s y)\left[\begin{array}{l}
r \\
s
\end{array}\right] \\
& =l \sum_{\left[\begin{array}{l}
r \\
s
\end{array}\right] \in S} e\left(\left[\begin{array}{l}
r \\
s
\end{array}\right]\right) \sum_{k=0}^{l-1} \frac{1}{2} B_{2}(r x / l+k r / l+s y)\left[\begin{array}{l}
r \\
s
\end{array}\right] \\
& =l \sum_{\left[\begin{array}{l}
r \\
s
\end{array}\right] \in S} \sum_{k=0}^{l-1} r_{\left[\begin{array}{l}
r_{s} \\
{[}
\end{array}\right]}(\phi((x+k) / l, y))\left[\begin{array}{l}
r \\
s
\end{array}\right] .
\end{aligned}
$$

On the other hand, (1.14) and (1.12) give

$$
\begin{aligned}
\Sigma_{2} & =l^{2} \sum_{\left[\begin{array}{l}
r \\
s
\end{array}\right] \in S^{\prime}} e\left(\left[\begin{array}{l}
r \\
s
\end{array}\right]\right) \frac{1}{2} B_{2}(r x / l+s y)\left[\begin{array}{l}
r \\
s
\end{array}\right] \\
& =l \sum_{k=0}^{l-1} \sum_{\left[\begin{array}{c}
r \\
s
\end{array}\right] \in S^{\prime}} r_{\left[\begin{array}{c}
r \\
s
\end{array}\right]}\left(\phi_{((x+k) / l, y)}\right)\left[\begin{array}{l}
r \\
s
\end{array}\right] .
\end{aligned}
$$

Combining (1.18), (1.20) and (1.21) gives $T_{l}^{*} \delta_{\Gamma}\left(\phi_{(x, y)}\right)=\delta_{\Gamma}\left(\phi_{(x, y)} \mid T_{l}\right)$. This proves Lemma 1.11.

Observe that since $\mathcal{P}(E)$ and $\mathcal{R}(E)$ are fractional ideals of $\mathbb{Q}\left[\epsilon_{1}, \epsilon_{2}\right], A_{E}$ and by duality $C_{E}$ have natural $\mathbb{Z}\left[\epsilon_{1}, \epsilon_{2}\right]$-module structures. The composite map (1.5) is a $\mathbb{Z}\left[\epsilon_{1}, \epsilon_{2}\right]$-module map with respect to these structures. If $x \in C_{E}$ is represented by $\eta\left(\delta_{\Gamma}(E)\right)$ with $\eta \in \mathcal{R}(E)^{*}$, then Lemma 1.11 shows that $T_{l}^{*} x$ is represented by $\eta\left(\delta_{\Gamma}\left(E \mid T_{l}\right)\right)=\eta\left(b_{l} \delta_{\Gamma}(E)\right)$, where $b_{l}$ is the eigenvalue of $T_{l}$ on $E$. Hence $T_{l}^{*}$ acts by multiplication by $b_{l}$ on $C_{E}$. 
Proposition 1.12. Under the same assumptions as in Proposition 1.9, the image of $C_{E}[p]_{\mathbb{F}_{p}}\left(\overline{\mathbb{F}}_{p}\right) \otimes_{\mathbb{F}_{p}} \overline{\mathbb{F}}_{p}$ under $\varphi$ is the $\overline{\mathbb{F}}_{p}$-module generated by $\bar{f}$.

Proof. Let $\eta \in \mathcal{R}(E)^{*}$. Choose $r \in \mathbb{Z}$ such that $r\left(\overline{\theta\left(\eta\left(\delta_{\Gamma}(E)\right)\right)}\right)$ is of order dividing $p$. Here $\overline{\theta(\cdot)}$ means the specialization to the special fiber of the $\mathcal{O}$-valued point of $C_{E / \mathbb{Z}_{p}}$ corresponding to $\theta(\cdot)$. By Prop. 1.2 and Lemma 1.11, we have for each prime $l$,

$$
\begin{aligned}
\left.\varphi\left(r \overline{\theta\left(\eta\left(\delta_{\Gamma}(E)\right)\right.}\right)\right) \mid T_{l} & \left.=\varphi\left(r T_{l}^{*} \overline{\theta\left(\eta\left(\delta_{\Gamma}(E)\right)\right.}\right)\right) \\
& =b_{l} \varphi\left(r \overline{\theta\left(\eta\left(\delta_{\Gamma}(E)\right)\right)}\right) \\
& =a_{l} \varphi\left(r \overline{\theta\left(\eta\left(\delta_{\Gamma}(E)\right)\right)}\right) .
\end{aligned}
$$

It follows that $\left.\varphi\left(r \overline{\theta\left(\eta\left(\delta_{\Gamma}(E)\right)\right.}\right)\right)=c \cdot \bar{f}$ for some $c \in \overline{\mathbb{F}}_{p}$. Since $C_{E}[p]_{/ \mathbb{F}_{p}}\left(\overline{\mathbb{F}}_{p}\right) \neq 0$ by Cor. 1.10 and $\eta \in \mathcal{R}(E)^{*}$ was arbitrary, $\varphi\left(C_{E}[p]_{/ \mathbb{F}_{p}}\left(\overline{\mathbb{F}}_{p}\right) \otimes \overline{\mathbb{F}}_{p}\right)=\overline{\mathbb{F}}_{p} \cdot \bar{f}$.

We can now complete the proof of Theorem 0.4. By Propositions 1.3, 1.12 and the injectivity of $\varphi$, we have $A_{f}[\mathfrak{p}]_{/ \mathbb{F}_{p}}\left(\overline{\mathbb{F}}_{p}\right) \otimes \overline{\mathbb{F}}_{p}=C_{E}[p]_{/ \mathbb{F}_{p}}\left(\overline{\mathbb{F}}_{p}\right) \otimes \overline{\mathbb{F}}_{p}$, hence $A_{f}[\mathfrak{p}]_{/ \mathbb{F}_{p}}\left(\overline{\mathbb{F}}_{p}\right)=C_{E}[p]_{/ \mathbb{F}_{p}}\left(\overline{\mathbb{F}}_{p}\right)$. Since the special fiber of $\left(A_{f}[\mathfrak{p}] \cap C_{E}\right)_{/ \mathbb{Z}_{p}}$ is $A_{f}[\mathfrak{p}]_{/ \mathbb{F}_{p}} \cap$ $C_{E / \mathbb{F}_{p}}$, it follows that $A_{f}[\mathfrak{p}] \cap C_{E} \neq 0$. This proves Theorem 0.4 .

\section{Cyclic isogenies of modular elliptic CuRves}

In this section, we prove Theorem 0.3. Let $A_{1}$ be an optimal curve over $\mathbb{Q}$ of conductor $N$. Let $p>2$ be a prime where $A_{1}$ has good ordinary reduction and let $\beta: A_{1} \rightarrow A$ be a cyclic $\mathbb{Q}$-isogeny of degree divisible by $p$. Let $\epsilon: G_{\mathbb{Q}} \rightarrow \operatorname{Aut}(\operatorname{ker} \beta[p])$ be the character giving the action of $G_{\mathbb{Q}}$ on $\operatorname{ker} \beta[p]$ and $\chi$ the Teichmüller character giving the action of $G_{\mathbb{Q}}$ on $\mu_{p}$. Consider the following three cases:

1. $\epsilon=1$,

2. $\epsilon=\chi$,

3. $\epsilon \neq 1, \chi$.

We shall show that if $p>7$, the first two cases do not occur and $\beta$ is étale at $p$ in the last case.

2.1. Reduction to $\epsilon \neq 1$, $\chi$. If $\epsilon=1$, then $\operatorname{ker} \beta[p] \subset A(\mathbb{Q})_{\text {tors }}$. By Mazur's classification theorem [11, Thm. 2], this implies $p \leq 7$. Thus if $p>7$, this case cannot occur.

Suppose next $\epsilon=\chi$, so $\operatorname{ker} \beta[p] \cong \mu_{p}$. Let $A^{\prime}=A_{1} / \operatorname{ker} \beta[p]$ and let $\beta^{\prime}: A_{1} \rightarrow A^{\prime}$ be the natural isogeny. By $[13, \S 15$, Thm. 1], the kernel of the dual isogeny $\check{\beta}^{\prime}: A^{\prime} \rightarrow A_{1}$ is the Cartier dual of $\operatorname{ker} \beta[p]$ and so is isomorphic to $\mathbb{Z} / p \mathbb{Z}$ as $G_{\mathbb{Q}^{-}}$ module. This implies that $p \mid \# A^{\prime}(\mathbb{Q})_{\text {tor. }}$ By Mazur's classification theorem again, this cannot happen if $p>7$.

We assume, henceforth, $\epsilon \neq 1, \chi$. Let $\rho: G_{\mathbb{Q}} \rightarrow \operatorname{Aut}\left(A_{1}[p]\right) \cong \mathrm{GL}_{2}\left(\mathbb{F}_{p}\right)$ be the Galois representation on the $p$-torsion points of $A_{1}$. Then with respect to a suitable basis, we have

$$
\rho \sim\left(\begin{array}{cc}
\epsilon & * \\
0 & \epsilon^{\prime}
\end{array}\right)
$$

for some character $\epsilon^{\prime}: G_{\mathbb{Q}} \rightarrow \mathbb{F}_{p}^{*}$. The Weil pairing shows that $\operatorname{det} \rho\left(\mathrm{Frob}_{l}\right) \equiv$ $l(\bmod p)$ for any prime $l \nmid N p$, where Frob $_{l}$ is a Frobenius element of $G_{\mathbb{Q}}$ for $l$. It 
follows from our assumption $\epsilon \neq 1, \chi$ that $\epsilon^{\prime} \neq 1$. Since $A_{1}$ is ordinary at $p$, there is an exact sequence of finite flat group schemes over $\mathbb{Z}_{p}$

$$
0 \rightarrow A_{1}[p]_{/ \mathbb{Z}_{p}}^{0} \rightarrow A_{1}[p]_{/ \mathbb{Z}_{p}} \rightarrow A_{1}[p]_{/ \mathbb{Z}_{p}}^{\text {ét }} \rightarrow 0
$$

where the flanking terms are each of order $p$ such that the inertia group $I_{p}$ acts via $\chi$ on the $G_{\mathbb{Q}_{p}}$-module associated to $A_{1}[p]_{/ \mathbb{Z}_{p}}^{0}$ and acts trivially on that associated to $A_{1}[p]_{/ \mathbb{Z}_{p}}^{\text {ét }}$. It follows that exactly one of $\epsilon$ and $\epsilon^{\prime}$ is unramifed at $p$. The next two lemmas show that we may assume $\epsilon^{\prime}$ is unramified at $p$.

Lemma 2.1. Let $\beta: A \rightarrow A^{\prime}$ be a cyclic $\mathbb{Q}$-isogeny of elliptic curves over $\mathbb{Q}$. Suppose $A$ has good reduction at $p$ and $\operatorname{ker} \beta\left[p^{\infty}\right](\overline{\mathbb{Q}})$ is unramified at $p$. Then $\beta$ is étale at $p$.

Proof. Let $\beta_{p}: A_{/ \mathbb{Z}_{p}} \rightarrow A_{/ \mathbb{Z}_{p}}^{\prime}$ be the extension of $\beta$ to Néron models over $\mathbb{Z}_{p}$. To show that $\beta_{p}$ is étale, we have to show that it is flat and unramified. Note that $\beta_{p}$ is quasi-finite and flat $[1,7.3$, Lemmas 1,2$]$. We check that it is unramified at points of residue characteristic $p$. The kernel $\operatorname{ker} \beta_{p}$ of $\beta_{p}$ is a finite flat group scheme over $\mathbb{Z}_{p}$. Let $K$ be the extension of $\mathbb{Q}_{p}$ cut out by $\operatorname{ker} \beta_{p}\left(\overline{\mathbb{Q}}_{p}\right)$ and let $\mathcal{O}$ denote its ring of integers. Since $A$ has good reduction at $p, A[m]$ is unramified at $p$ for all $m$ prime to $p$ by the Néron-Ogg-Shafarevich criterion. By the assumption on $\operatorname{ker} \beta\left[p^{\infty}\right](\overline{\mathbb{Q}})$, $\operatorname{ker} \beta\left(\overline{\mathbb{Q}}_{p}\right)$ is unramified as $G_{\mathbb{Q}_{p}}$-module. Thus $\operatorname{ker} \beta_{p}$ is a Néron model of $\operatorname{ker} \beta_{p / \mathbb{Q}_{p}}$ ([1, 7.1, Cor. 6]). Let $x \in \operatorname{ker} \beta_{p}(\mathcal{O})$ be the $\mathcal{O}$-valued point corresponding to a generator of $\operatorname{ker} \beta_{p}(K)$. By the specialization lemma in $[11, \S 1]$, the order of the specialization of $x$ to the residue field of $\mathcal{O}$ equals the order of $x$. So if $\bar{\beta}_{p}$ is the reduction of $\beta_{p}$ mod $p$, then

$$
\# \operatorname{ker} \bar{\beta}_{p}=\# \operatorname{ker} \beta=\operatorname{deg} \beta=\operatorname{deg} \bar{\beta}_{p} .
$$

By $[20,4.10(\mathrm{a})], \# \operatorname{ker} \bar{\beta}_{p}$ equals the degree of separability $\operatorname{deg}_{s} \bar{\beta}_{p}$ of $\bar{\beta}_{p}$. $\operatorname{So} \operatorname{deg}_{s} \bar{\beta}_{p}=$ $\operatorname{deg} \bar{\beta}_{p}$; hence $\bar{\beta}_{p}$ is separable and unramified by $[20,4.10(\mathrm{c})]$. This proves that $\beta_{p}$ is étale.

Lemma 2.2. Let $\beta: A \rightarrow A^{\prime}$ be a cyclic $\mathbb{Q}$-isogeny of degree $d$ divisible by $p$. Suppose $A$ has good ordinary reduction at $p$ and $\operatorname{ker} \beta[p]$ is unramified at $p$. Then $\operatorname{ker} \beta\left[p^{\infty}\right]$ is unramified at $p$.

Proof. Let $\epsilon_{1}$ be the Galois character on $\operatorname{ker} \beta\left[p^{\infty}\right]$. Write $\epsilon_{1} \oplus \epsilon_{2}$ for the semisimplification of the Galois representation $\rho: G_{\mathbb{Q}} \rightarrow \operatorname{Aut}\left(A\left[p^{r}\right]\right)$, where $\epsilon_{2}$ is some Galois character and $p^{r} \| d$. Since $p$ is ordinary, one of the characters $\epsilon_{1}$ and $\epsilon_{2}$ is unramified at $p$ and the other when restricted to $I_{p}$ is the cyclotomic character on $\mu_{p^{r}}$. By our assumption, $\epsilon_{1}(\bmod p)$ is unramified at $p$. Since $p>2, \epsilon_{1}$ is unramified at $p$. This proves the lemma.

If $\epsilon$ is unramified at $p$, then by Lemma $2.2, \operatorname{ker} \beta\left[p^{\infty}\right]$ is unramified at $p$. Thus $\beta$ is étale at $p$ by Lemma 2.1, hence Theorem 0.3. So we assume, from now on,

$\epsilon$ is ramified at $p$.

Let $\pi: X_{1}(N) \rightarrow A_{1}$ be a modular parametrization and let $f$ be the associated weight 2 normalized newform on $\Gamma_{1}(N)$. Write

$$
f(z)=\sum_{n=1}^{\infty} a_{n} q^{n}, \quad q=e^{2 \pi i z}, \quad z \in \mathfrak{H}
$$


for the $q$-expansion of $f$ at the cusp $\infty$. We show that there is a weight 2 Eisenstein series $E$ on $\Gamma_{1}(N)$ associated to $\rho$ such that $\mathfrak{P}$ is an Eisenstein prime for $E$ and $f$ (Prop. 2.6). Applying Theorem 0.4 and the classification theorem of rational cyclic isogenies of elliptic curves over $\mathbb{Q}$, we deduce that $p \leq 5(\S 2.3)$.

2.2. Eisenstein series associated to $\rho$. Let $\chi_{1}$ and $\chi_{2}$ be two Dirichlet characters, not necessarily primitive, modulo $N_{1}$ and $N_{2}$ respectively. Put

$$
G\left(a_{1}, a_{2} ; \mathfrak{a}_{1}, \mathfrak{a}_{2}\right)=\frac{1}{N_{2}} \sum_{t \in N_{2}^{-1} \mathbb{Z} / \mathbb{Z}} e^{2 \pi i\left(-t a_{2}\right)} E\left(z ; a_{1}, t ; \mathfrak{a}_{1}, \mathfrak{a}_{2}^{-1}\right)
$$

and

$$
E\left(\chi_{1}, \chi_{2}\right)=\frac{1}{2} \sum_{a_{1}=0}^{N_{1}-1} \sum_{a_{2}=0}^{N_{2}-1} \chi_{1}\left(a_{1}\right) \chi_{2}\left(a_{2}\right) G\left(a_{1}, a_{2} ; N_{1} \mathbb{Z}, \mathbb{Z}\right)
$$

where $E\left(z ; a_{1}, a_{2} ; \mathfrak{a}_{1}, \mathfrak{a}_{2}\right)$ is as in (1.7).

Proposition 2.3. Notation being as above, assume that not both $\chi_{1}$ and $\chi_{2}$ are the trivial character of any conductor. Then

(a) $E\left(\chi_{1}, \chi_{2}\right)$ is a weight 2 Eisenstein series on $\Gamma_{0}\left(N_{1} N_{2}\right)$ of character $\left(\chi_{1} \chi_{2}\right)^{-1}$.

(b) The Dirichlet series $L(E, s):=\sum_{n=1}^{\infty} b_{n} n^{-s}$ of $E\left(\chi_{1}, \chi_{2}\right)$ is

$$
L\left(\chi_{1}, s\right) L\left(\chi_{2}, s-1\right) \text {, }
$$

where $b_{n}$ is the $n$-th Fourier coefficient of $E\left(\chi_{1}, \chi_{2}\right)$ at $\infty$.

(c) If $\chi_{1} \neq 1$, then the constant term of the Fourier expansion of $E\left(\chi_{1}, \chi_{2}\right)$ at $\infty$ is 0 .

Proof. (a) and (b) follow from [19, Prop. 3.4] for Artin characters of degree 1 of totally real number fields of degree $>1$. But, as remarked in $[25, \S 1.5]$, the same result holds for $\mathbb{Q}$ if $\chi_{1}$ and $\chi_{2}$ are not both the trivial character of any conductor. (c) follows from the Fourier expansion of $E\left(z ; a_{1}, a_{2} ; N_{1} \mathbb{Z}, \mathbb{Z}\right)$ in (1.8), the definition of $E\left(\chi_{1}, \chi_{2}\right)$ in (2.4), and the assumption that $\chi_{1} \neq 1$.

Remark 2.4. Using (1.8), we find that the Fourier expansion of $E\left(\chi_{1}, \chi_{2}\right)$ at $\infty$ is

$$
E\left(\chi_{1}, \chi_{2}\right)(z)=\sum_{c=1}^{\infty} \sum_{b=1}^{\infty} \chi_{1}(c) \chi_{2}(b) b e^{2 \pi i(b c z)} .
$$

From this, we can deduce Prop. 2.3(b) in a similar fashion to [21, Prop. 3.4.2(b)].

We want to apply Prop. 2.3 to certain Dirichlet characters associated to $\epsilon$ and $\epsilon^{\prime}$ in (2.1) to get an Eisenstein series with suitable properties. For this, we consider the ramification behavior of the primes dividing $N$ in $\mathbb{Q}\left(A_{1}[p]\right) / \mathbb{Q}$. For each prime $q$, let $T_{q}$ be the $q$-adic Tate module of $A_{1}$ and $V_{q}=T_{q} \otimes_{\mathbb{Z}_{q}} \mathbb{Q}_{q}$. Let $H_{q}=H_{\text {ét }}^{1}\left(A_{1} \times_{\mathbb{Q}} \overline{\mathbb{Q}}, \mathbb{Q}_{q}\right)$. Then $V_{q}=H_{q} \otimes_{\mathbb{Q}_{q}} \mathbb{Q}_{q}(1)$, and the collection $\left\{H_{q}\right\}_{q}$ forms a compatible system $V$ of $q$-adic representations of $G_{\mathbb{Q}}$ whose $L$-function $L(V, s)$ is defined by an Euler product (for $\operatorname{Re} s>\frac{3}{2}$ ):

$$
L(V, s)=\prod_{l} L_{l}(V, s)=\prod_{l} \operatorname{det}\left(1-\operatorname{Frob}_{l}^{-1} l^{-s} \mid\left(H_{q}\right)^{I_{l}}\right)^{-1},
$$

where for each $l, q$ is a prime $\neq l$ and $\left(H_{q}\right)^{I_{l}}$ is the maximal subspace of $H_{q}$ on which $I_{l}$ acts trivially. 
Let $l$ be a prime dividing $N$. Suppose $l \| N$. Then $A_{1}$ has multiplicative reduction at $l$. By $[16, \S 1.12]$, there is an unramified extension $K_{l} / \mathbb{Q}_{l}$ of degree $\leq 2$ such that $A_{1}$ is isomorphic over $K_{l}$ to the Tate curve $E_{q}=\mathbb{G}_{m} / q^{\mathbb{Z}}$ for some $q \in l \mathbb{Z}_{l}$ determined by the $j$-invariant of $A_{1}$. We have an exact sequence of $G_{K_{l}}$-modules for each $n$ :

$$
0 \rightarrow \mu_{p^{n}} \rightarrow A_{1}\left[p^{n}\right] \rightarrow \mathbb{Z} / p^{n} \mathbb{Z} \rightarrow 0 .
$$

As $\rho$ is given by (2.1), both $\epsilon$ and $\epsilon^{\prime}$ are unramified at $l$. On taking inverse limit over $n$ and then tensoring with $\mathbb{Q}_{p},(2.6)$ gives a (non-split) exact sequence of $G_{K_{l}}$-modules:

$$
0 \rightarrow \mathbb{Q}_{p}(1) \rightarrow V_{p} \rightarrow \mathbb{Q}_{p} \rightarrow 0 .
$$

Thus $G_{\mathbb{Q}_{l}}$ acts via some character $\psi_{l}$ of $\operatorname{Gal}\left(K_{l} / \mathbb{Q}_{l}\right)$ on $\left(V_{p}\right)_{I_{l}}$ (= the maximal quotient of $V_{p}$ on which $I_{l}$ acts trivially). From $(2.1),\left.\psi_{l} \equiv \epsilon\right|_{G_{\mathbb{Q}_{l}}}(\bmod p)$ or $\left.\psi_{l} \equiv \epsilon^{\prime}\right|_{G_{\mathbb{Q}_{l}}}(\bmod p)$. Let $S_{1}=\left\{l: l \| N,\left.\psi_{l} \equiv \epsilon\right|_{G_{\mathbb{Q}_{l}}}(\bmod p)\right\}$ and $S_{2}=\{l$ : $\left.l \| N, l \notin S_{1}\right\}$.

Suppose now $l^{2} \mid N$. Then $A_{1}$ has additive reduction at $l$. By [16, $\S 5.6$ Prop. 23b], the images of $I_{l}$ under $\epsilon$ and $\epsilon^{\prime}$ are cyclic of order 2, 3, 4 or 6. In particular, $\epsilon$ and $\epsilon^{\prime}$ are ramified at $l$.

Let $f_{\epsilon_{0, \text { prim }}}$ and $f_{\epsilon_{0, \text { prim }}^{\prime}}$ be the conductors of the primitive Dirichlet characters $\epsilon_{0, \text { prim }}$ and $\epsilon_{0, \text { prim }}^{\prime}$ associated to $\epsilon$ and $\epsilon^{\prime}$ respectively. Let $\epsilon_{0, p r i m} \chi^{-1}$ be the primitive Dirichlet character such that $\epsilon_{0, \text { prim }}=\epsilon_{0, \text { prim }} \chi^{-1} \cdot \chi$. Here we view $\chi$ as both a character of $G_{\mathbb{Q}}$ and a Dirichlet character. Since $\epsilon^{\prime}$ is unramified at $p$ (cf. (2.2)) and $\epsilon(l) \epsilon^{\prime}(l) \equiv l(\bmod p)$ for any $l \nmid N p$, the conductor $f_{\epsilon_{0, \operatorname{prim}} \chi^{-1}}$ of $\epsilon_{0, \operatorname{prim}} \chi^{-1}$ is prime to $p$. Now let $\epsilon_{0} \chi^{-1}$ (resp. $\epsilon_{0}, \epsilon_{0}^{\prime}$ ) be the Dirichlet character modulo $f_{\epsilon_{0} \chi^{-1}}:=f_{\epsilon_{0, p r i m} \chi^{-1}} \cdot \prod_{l \in S_{2}} l$ (resp. $f_{\epsilon_{0}}:=f_{\epsilon_{0, \text { prim }}} \cdot \prod_{l \in S_{2}} l, f_{\epsilon_{0}^{\prime}}:=f_{\epsilon_{0, \text { prim }}^{\prime}} \cdot \prod_{l \in S_{1}} l$ ) whose primitive character is $\epsilon_{0, \text { prim }} \chi^{-1}$ (resp. $\epsilon_{0, \text { prim }}, \epsilon_{0, \text { prim }}^{\prime}$ ). (For a Dirichlet character $\psi$ modulo $m$, we set $\psi(n)=0$ if $(n, m) \neq 1$.)

Lemma 2.5. (a) $f_{\epsilon_{0} \chi^{-1}} f_{\epsilon_{0}^{\prime}}$ divides $N$.

(b) $f_{\epsilon_{0} \chi^{-1}} f_{\epsilon_{0}^{\prime}}$ and $N$ have the same prime divisors.

Proof. (a) By a result of Carayol [2, 0.8], the level $N$ of $f$ is equal to the conductor of the $p$-adic representation $V_{p}$. (For the definition of the latter, see for example [4, $\S 1.1]$.) Put $\Phi=\operatorname{ker} \beta[p]$ and $\Phi^{\prime}=A_{1}[p] / \Phi$. For any prime $l \neq p$, we have

$$
\operatorname{dim}_{\mathbb{F}_{p}} A_{1}[p]^{G_{i}} \leq \operatorname{dim}_{\mathbb{F}_{p}} \Phi^{G_{i}}+\operatorname{dim}_{\mathbb{F}_{p}} \Phi^{\prime G_{i}},
$$

where $G_{0} \supset G_{1} \supset \cdots$ is the series of ramification groups in $\operatorname{Gal}\left(\mathbb{Q}_{l}\left(A_{1}[p]\right) / \mathbb{Q}_{l}\right)$. Since $\sum_{i=0}^{\infty}\left[G_{0}: G_{i}\right]^{-1} \operatorname{dim}_{\mathbb{F}_{p}}\left(\Phi / \Phi^{G_{i}}\right)$ is the $l$-part of $f_{\epsilon_{0, \text { prim }}}$ and similarly for $\Phi^{\prime}$ and $\epsilon^{\prime}$, the $l$-part of $f_{\epsilon_{0, \text { prim }}} f_{\epsilon_{0, p r i m}^{\prime}}$ divides $N$. To see that the $l$-part of $f_{\epsilon_{0}} f_{\epsilon_{0}^{\prime}}$ divides $N$, we need only consider the case $l \| N$. In this case $l \nmid f_{\epsilon_{0, p r i m}} f_{\epsilon_{0}^{\prime} \text { prim }}$, so by the definition of $f_{\epsilon_{0}}$ and $f_{\epsilon_{0}^{\prime}}$ we have $l \| f_{\epsilon_{0}} f_{\epsilon_{0}^{\prime}}$. Hence $f_{\epsilon_{0} \chi^{-1}} f_{\epsilon_{0}^{\prime}}$ divides $N$. This proves (a).

(b) Let $l$ be a prime. If $l \| N$, then $l \mid f_{\epsilon_{0}}$ or $l \mid f_{\epsilon_{0}^{\prime}}$. If $l^{2} \mid N$, then $\epsilon$ and $\epsilon^{\prime}$ are both ramified at $l$, so $l \mid f_{\epsilon_{0}}$ and $l \mid f_{\epsilon_{0}^{\prime}}$. This proves (b).

We now apply Prop. 2.3 to $\chi_{1}=\epsilon_{0}^{\prime}$ and $\chi_{2}=\epsilon_{0} \chi^{-1}$ to get an Eisenstein series $E=E\left(\epsilon_{0}^{\prime}, \epsilon_{0} \chi^{-1}\right)$. (Recall that, under our assumption (2.2), $\epsilon_{0}^{\prime}$ and $\epsilon_{0} \chi^{-1}$ are both non-trivial.) 
Proposition 2.6. The Fourier coefficients of the q-expansions of $E$ and $f$ at $\infty$ are congruent mod $\mathfrak{P}$ :

$$
E(q) \equiv f(q) \quad(\bmod \mathfrak{P})
$$

Proof. By Prop. 2.3(b), the Euler factor $L_{l}(E, s)$ of $L(E, s)$ at a prime $l$ is $\left(1-\epsilon_{0}^{\prime}(l) l^{-s}\right)^{-1}\left(1-\epsilon_{0} \chi^{-1}(l) l^{1-s}\right)^{-1}$. We have a formal Euler product for the Dirichlet series $L(f, s)$ of $f$ :

$$
L(f, s)=\prod_{l} L_{l}(f, s)=\prod_{l \mid N}\left(1-a_{l} l^{-s}\right)^{-1} \prod_{l \nmid N}\left(1-a_{l} l^{-s}+l^{1-2 s}\right)^{-1},
$$

where $a_{l}$ is the $l$-th Fourier coefficient of $f$ in $(2.3)$. By [2], $L(f, s)=L(V, s)$.

For $l \nmid N p$, we have $l \epsilon_{0} \chi^{-1}(l) \cdot \epsilon_{0}^{\prime}(l)=l$ and, by the Eichler-Shimura relations,

$$
a_{l} \equiv \operatorname{tr}\left(\rho\left(\operatorname{Frob}_{l}\right)\right)=\epsilon\left(\operatorname{Frob}_{l}\right)+\epsilon^{\prime}\left(\operatorname{Frob}_{l}\right) \equiv l \epsilon_{0} \chi^{-1}(l)+\epsilon_{0}^{\prime}(l) \quad(\bmod \mathfrak{P}),
$$

So

$\left(1-a_{l} l^{-s}+l^{1-2 s}\right)^{-1} \equiv\left(1-l \epsilon_{0} \chi^{-1}(l) l^{-s}\right)^{-1}\left(1-\epsilon_{0}^{\prime}(l) l^{-s}\right)^{-1}=L_{l}(E, s) \quad(\bmod \mathfrak{P})$.

Let $l \mid N$. Then $L_{l}(f, s)=\left(1-a_{l} l^{-s}\right)^{-1}$. Suppose $l \| N$. By (2.1) and the definition of $\epsilon_{0}^{\prime}$ and $\epsilon_{0} \chi^{-1}$, we have

$$
\left(1-a_{l} l^{-s}\right)^{-1} \equiv\left(1-\epsilon^{*}(l) l^{-s}\right)^{-1} \equiv L_{l}(E, s) \quad(\bmod \mathfrak{P}),
$$

where $\epsilon^{*}=\epsilon_{0}$ or $\epsilon_{0}^{\prime}$ according as $l \in S_{1}$ or $l \in S_{2}$. Next suppose $l^{2} \mid N$. Then $a_{l}=0$ and $L_{l}(f, s)=1$. Since $\epsilon_{0}^{\prime}(l)=\epsilon_{0} \chi^{-1}(l)=0, L_{l}(E, s)=1$.

Finally, suppose $l=p$. Since $A_{1}$ is ordinary at $p, a_{p}$ is congruent mod $\mathfrak{P}$ to the eigenvalue of Frobenuis on the $p$-adic Tate module of $A_{1 / \mathbb{F}_{p}}$. So we have

$$
\left(1-a_{p} p^{-s}+p \cdot p^{-2 s}\right)^{-1} \equiv\left(1-a_{p} p^{-s}\right)^{-1} \equiv\left(1-\epsilon_{0}^{\prime}(p) p^{-s}\right)^{-1} \quad(\bmod \mathfrak{P}) .
$$

Hence $L_{l}(f, s)$ is congruent $\bmod \mathfrak{P}$ to $L_{l}(E, s)$ for each $l$. This shows that $a_{n} \equiv$ $b_{n}(\bmod \mathfrak{P})$ for each $n \geq 1$ and, together with Prop. 2.3(c), proves the proposition.

We show that $E=E\left(\epsilon_{0}^{\prime}, \epsilon_{0} \chi^{-1}\right)$ is a common eigenfunction for all $T_{l}$ and $\langle l\rangle$.

Lemma 2.7. For any prime $l$, we have

(a) $E \mid\langle l\rangle=E$ if $l \nmid N$;

(b) $E \mid T_{l}=\left(\epsilon_{0}^{\prime}(l)+l \epsilon_{0} \chi^{-1}(l)\right) E$.

Proof. (a) Since $\epsilon_{0}^{\prime}(l) \cdot \epsilon_{0} \chi^{-1}(l)=1$ for all $l \nmid N, E$ is modular on $\Gamma_{0}(N)$ by Prop. $2.3\left(\right.$ a). Hence $E|\langle l\rangle=E| \sigma_{l}=E$.

(b) We use the $q$-expansion of $E$ at $\infty$ given by (2.5):

$$
\begin{aligned}
E(z) & =\sum_{c=1}^{\infty} \sum_{b=1}^{\infty} \epsilon_{0}^{\prime}(c) \epsilon_{0} \chi^{-1}(b) b q^{b c}, \quad q=e^{2 \pi i z}, \\
& =\sum_{n=1}^{\infty} b_{n} q^{n},
\end{aligned}
$$

where $b_{n}=\sum_{b c=n} \epsilon_{0}^{\prime}(c) \epsilon_{0} \chi^{-1}(b) b$ for each $n \geq 1$. 
By Prop. 2.3(a), the level of $E$ divides $f_{\epsilon_{0} \chi^{-1}} f_{\epsilon_{0}^{\prime}}$, which has the same prime divisors as $N$ by Lemma 2.5. The action of $T_{l}$ on $E$ is then given by $([18,(3.5 .12)])$ :

$$
\begin{aligned}
E \mid T_{l} & =\sum_{n=1}^{\infty} b_{l n} q^{n}+l \sum_{n=1}^{\infty} b_{n} q^{l n}, \quad l \nmid N, \\
& =\sum_{n=1}^{\infty} b_{l n} q^{n}, \quad l \mid N .
\end{aligned}
$$

Suppose first $l \nmid N$. Then

$$
\begin{aligned}
b_{l n} & =\sum_{b c=l n} \epsilon_{0}^{\prime}(c) \epsilon_{0} \chi^{-1}(b) b \\
& =\sum_{\substack{b c=l n \\
l \nmid b}} \epsilon_{0}^{\prime}(c) \epsilon_{0} \chi^{-1}(b) b+\sum_{\substack{b c=l n \\
l \mid b}} \epsilon_{0}^{\prime}(c) \epsilon_{0} \chi^{-1}(b) b \\
& =\epsilon_{0}^{\prime}(l) \sum_{\substack{b c^{\prime}=n \\
l \nmid b}} \epsilon_{0}^{\prime}\left(c^{\prime}\right) \epsilon_{0} \chi^{-1}(b) b+l \epsilon_{0} \chi^{-1}(l) \sum_{b^{\prime} c=n} \epsilon_{0}^{\prime}(c) \epsilon_{0} \chi^{-1}\left(b^{\prime}\right) b^{\prime} .
\end{aligned}
$$

Since $\epsilon_{0}^{\prime}(l) \epsilon_{0}(l)=\chi(l)$ for all $l \nmid N p$ and $p \nmid f_{\epsilon_{0}^{\prime}} f_{\epsilon_{0} \chi^{-1}}$, it follows that $\epsilon_{0}^{\prime}(l)=$ $\epsilon_{0} \chi^{-1}\left(l^{-1}\right)$ for all $l \nmid N$. So

$$
\begin{aligned}
l b_{n} & =l \sum_{b c=n} \epsilon_{0}^{\prime}(c) \epsilon_{0} \chi^{-1}(b) b \\
& =\epsilon_{0} \chi^{-1}\left(l^{-1}\right) \sum_{b c=n} \epsilon_{0}^{\prime}(c) \epsilon_{0} \chi^{-1}(l b) l b \\
& =\epsilon_{0} \chi^{-1}\left(l^{-1}\right) \sum_{\substack{b c=l n \\
l \mid b}} \epsilon_{0}^{\prime}(c) \epsilon_{0} \chi^{-1}(b) b \\
& =\epsilon_{0}^{\prime}(l) \sum_{\substack{b c=l n \\
l \mid b}} \epsilon_{0}^{\prime}(c) \epsilon_{0} \chi^{-1}(b) b .
\end{aligned}
$$

(b) follows from (2.7), (2.9) and (2.10) for $l \nmid N$. For $l \mid N$, we have

$$
b_{l n}= \begin{cases}l \epsilon_{0} \chi^{-1}(l) b_{n} & \text { if } l \mid f_{\epsilon_{0}^{\prime}} \text { and } l \nmid f_{\epsilon_{0} \chi^{-1}}, \\ \epsilon_{0}^{\prime}(l) b_{n} & \text { if } l \mid f_{\epsilon_{0} \chi^{-1}} \text { and } l \nmid f_{\epsilon_{0}^{\prime}}, \\ 0 & \text { otherwise, }\end{cases}
$$

and (b) follows from (2.8).

2.3. Completion of proof of Theorem $\mathbf{0 . 3}$ in the case $\epsilon \neq 1, \chi$. We can now complete the proof of Theorem 0.3 in this case, assuming (2.2). Applying Theorem 0.4 to $f$ and $E$, we have $A_{1}[p] \cap C_{E} \neq 0$. By [21, Thm. 3.2.4], $C_{E}$ is stable under the action of $G_{\mathbb{Q}}$, which is given by $\epsilon^{\prime}$. Since $\epsilon$ (resp. $\epsilon^{\prime}$ ) is ramified (resp. unramified) at $p$, it follows that $\operatorname{ker} \beta[p]$ and $A_{1}[p] \cap C_{E}$ are two independent cyclic subgroups of order $p$ defined over $\mathbb{Q}$. Hence $A_{1} / \operatorname{ker} \beta[p]$ is an elliptic curve over $\mathbb{Q}$ which has a cyclic subgroup of order $p^{2}$ defined over $\mathbb{Q}$. Kenku [7, Thm. 1] has shown that the table in the introduction of [11] is a complete list of $d$ for which there is a rational cyclic $d$-isogeny of elliptic curves over $\mathbb{Q}$. This implies that $p \leq 5$. Thus if $p>7, \epsilon$ is unramified at $p$. This completes the proof of Theorem 0.3 . 


\section{INTEGRALITY OF $p$-ADIC $L$-FUNCTIONS}

We now apply Theorem 0.3 to establish Theorem 0.2. We shall use the following result of Stevens.

Theorem 3.1. ([23, Thm. 4.6]) Suppose $p>2$. Let $\pi: X_{1}(N) \rightarrow A$ be a modular parametrization and $c(\pi)$ the Manin constant of $\pi$. Then $c(\pi) \nu_{A, \Delta}$ takes values in $\mathcal{L}(A) \otimes \mathbb{Z}_{p}$

To prove Theorem 0.2 , it suffices by Theorem 3.1 to show that there is a modular parametrization $\pi: X_{1}(N) \rightarrow A$ such that $c(\pi)$ is a $p$-unit. Let $\mathcal{A}$ be the $\mathbb{Q}$-isogeny class of elliptic curves over $\mathbb{Q}$ containing $A$. Let $A_{1}$ be the optimal curve in $\mathcal{A}$ and $\pi_{1}: X_{1}(N) \rightarrow A_{1}$ an optimal parametrization (cf. the introduction). Let $n$ be the largest square dividing $N$.

Proposition 3.2. With notation as above, $c\left(\pi_{1}\right) \in \mathbb{Z}[1 / 2 n]^{*}$.

Proof. The analogous result for a strong parametrization $\pi_{0}: X_{0}(N) \rightarrow A_{0}$ (which takes the cusp $\infty$ to the origin of $A_{0}$ ) of the strong Weil curve $A_{0} \in \mathcal{A}$ has been proved in $\left[11\right.$, Cor. 4.1] by showing that $\pi_{0}: X_{0}(N)_{/ \mathbb{Z}\left[\frac{1}{2 n}\right]}^{\mathrm{smooth}} \rightarrow A_{0 / \mathbb{Z}\left[\frac{1}{2 n}\right]}$ is a formal immersion along the $\infty$-section, where $X_{0}(N)_{\mathbb{Z}\left[\frac{1}{2 n}\right]}^{\operatorname{smooth}}$ is the smooth locus of $X_{0}(N)_{/ \mathbb{Z}\left[\frac{1}{2 n}\right]} \rightarrow$ Spec $\mathbb{Z}$. An analysis of the arguments used there shows that for an optimal parametrization $\pi_{1}: X_{1}(N) \rightarrow A_{1}$ which takes the cusp 0 to the origin of $A_{1}, \pi_{1}: X_{1}(N)_{/ \mathbb{Z}\left[\frac{1}{2 n}\right]}^{\text {smooth }} \rightarrow A_{1 / \mathbb{Z}\left[\frac{1}{2 n}\right]}$ is a formal immersion along the 0section. Since $X_{1}(N)_{/ \overline{\mathbb{F}}_{l}}$ is irreducible if $l \nmid N$ and since the Atkin-Lehner involution $w_{N}$ interchanges the two irreducible components of $X_{1}(N)_{/ \overline{\mathbb{F}}_{l}}$ if $l \| N$, we have $c\left(\pi_{1}\right) \in \mathbb{Z}\left[\frac{1}{2 n}\right]^{*}$.

Lemma 3.3. Let $X \stackrel{f}{\rightarrow} Y, Y \stackrel{g}{\rightarrow} Z$ be morphisms of schemes. Suppose that $f$ is smooth. Then there is an exact sequence of $\mathcal{O}_{X}$-modules

$$
0 \rightarrow f^{*} \Omega_{Y / Z}^{1} \rightarrow \Omega_{X / Z}^{1} \rightarrow \Omega_{X / Y}^{1} \rightarrow 0
$$

where $\Omega_{X / Y}^{1}$ is the sheaf of relative differentials of degree 1 of $X$ over $Y$ and similarly for $\Omega_{Y / Z}^{1}$ and $\Omega_{X / Z}^{1}$, and $X$ is considered as a $Z$-scheme via $g \circ f$.

Proof. See [1, 2.2 Prop. 5(b)] and the remark after it.

Corollary 3.4. Let $\beta: A \rightarrow A^{\prime}$ be a $\mathbb{Q}$-isogeny of elliptic curves over $\mathbb{Q}$ étale at p. Let $\Omega_{A / \mathbb{Z}_{p}}^{1}$ and $\Omega_{A^{\prime} / \mathbb{Z}_{p}}^{1}$ be the sheaves of Néron differentials on $A_{/ \mathbb{Z}_{p}}$ and $A_{/ \mathbb{Z}_{p}}^{\prime}$ respectively. Then $\beta$ induces an isomorphism

$$
\beta^{*}: H^{0}\left(A_{/ \mathbb{Z}_{p}}^{\prime}, \Omega_{A^{\prime} / \mathbb{Z}_{p}}^{1}\right) \stackrel{\sim}{\rightarrow} H^{0}\left(A_{/ \mathbb{Z}_{p}}, \Omega_{A / \mathbb{Z}_{p}}^{1}\right) .
$$

Proof. Since $\beta$ is étale at $p, \beta_{/ \mathbb{Z}_{p}}$ is smooth and $\Omega_{A_{/ \mathbb{Z}_{p} / A^{\prime} \mathbb{Z}_{p}}^{1}}=0$. So the exact sequence in Lemma 3.3 gives $\beta^{*} \Omega_{A^{\prime} / \mathbb{Z}_{p}}^{1} \cong \Omega_{A / \mathbb{Z}_{p}}^{1}$. Hence $H^{0}\left(A_{/ \mathbb{Z}_{p}}^{\prime}, \Omega_{A^{\prime} / \mathbb{Z}_{p}}^{1}\right) \cong$ $H^{0}\left(A_{/ \mathbb{Z}_{p}}, \beta^{*} \Omega_{A^{\prime} / \mathbb{Z}_{p}}^{1}\right) \cong H^{0}\left(A_{/ \mathbb{Z}_{p}}, \Omega_{A / \mathbb{Z}_{p}}^{1}\right)$.

We can now prove Theorem 0.2. As remarked above, it suffices to show that there is a modular parametrization $\pi: X_{1}(N) \rightarrow A$ such that $c(\pi)$ is a $p$-unit. We show that a modular parametrization $\pi: X_{1}(N) \rightarrow A$ of minimal degree meets this requirement. By the definition of optimality, there is a $\mathbb{Q}$-isogeny $\beta: A_{1} \rightarrow A$ 
such that $\pi=\beta \circ \pi_{1}$. We have $\operatorname{deg} \pi=\operatorname{deg} \beta \operatorname{deg} \pi_{1}$. It follows that $\operatorname{deg} \beta$ is minimal among all isogenies from $A_{1}$ to $A$. Thus $\beta$ must be cyclic. Let $\omega_{A_{1}}$ and $\omega_{A}$ be Néron differentials on $A_{1}$ and $A$ respectively, and let $c(\beta) \in \mathbb{Z}$ be such that $\beta^{*} \omega_{A}=c(\beta) \omega_{A_{1}}$. If $p \nmid \operatorname{deg} \beta$, then $p \nmid c(\beta)$. If $p \mid \operatorname{deg} \beta$, then $\beta$ is étale at $p$ by Theorem 0.3 , and so $p \nmid c(\beta)$ by Corollary 3.4. Since $c(\pi)=c(\beta) c\left(\pi_{1}\right)$ and $c\left(\pi_{1}\right)$ is a $p$-unit by Prop. 3.2, $c(\pi)$ is a $p$-unit. This completes the proof of Theorem 0.2.

\section{REFERENCES}

1. S. Bosch, W. Lütkebohmert and M. Raynaud, Néron Models, Ergeb. der Math. und ihrer Grenzgeb., 3. Folge, Bd. 21, Springer-Verlag, Berlin, 1990. MR 91i:14034

2. H. Carayol, Sur les représentations l-adiques associées aux formes modulaires de Hilbert, Ann. Sci. Éc. Norm. Sup., IV Sér. 19 (1986), 409-468. MR 89c:10083

3. P. Cartier, Une nouvelle opération sur les formes differentielles, C. R. Acad. Sc. Paris 244 (1957), 426-428. MR 18:870b

4. J. Coates and C.-G. Schmidt, Iwasawa theory for the symmetric square of an elliptic curve, J. Reine Angew. Math. 375 (1987), 104-156. MR 88i:11077

5. P. Deligne and M. Rapoport, Les schémas de modules de courbes elliptiques, Lecture Notes in Math., vol 349, Springer-Verlag, Berlin, 1973, pp. 143-316. MR 49:2762

6. H. Hida, On congruence divisors of cusp forms as factors of the special values of their zeta functions, Invent. Math. 64 (1981), 221-262. MR 83h:10066

7. M.A. Kenku, On the number of $\mathbb{Q}$-isomorphism classes of elliptic curves in each $\mathbb{Q}$-isogeny class, J. Number Theory 15 (1982), 199-202. MR 84c:14036

8. Y. Manin, Parabolic points and zeta functions of modular curves, Izv. Akad. Nauk SSSR Ser. Mat. 36 (1972), 19-65 (Russian); English transl. in Math. USSR-Izv. 6 (1972), 19-64. MR 47:3396

9. B. Mazur and P. Swinnerton-Dyer, Arithmetic of Weil curves, Invent. Math. 25 (1974), 1-61. MR 50:7152

10. B. Mazur, Modular curves and the Eisenstein ideal, Inst. Hautes Études Sci. Publ. Math. 47 (1977), 33-186. MR 80c: 14015

11. B. Mazur, Rational isogenies of prime degree, Invent. Math. 44 (1978), 129-162. MR 80h: 14022

12. B. Mazur, J. Tate and J. Teitelbaum, On p-adic analogues of the conjectures of Birch and Swinnerton-Dyer, Invent. Math. 84 (1986), 1-48. MR 87e:11076

13. D. Mumford, Abelian Varieties, Oxford Univ. Press, Oxford, 1970. MR 44:219

14. K.A. Ribet, On modular representations of $\mathrm{Gal}(\overline{\mathbb{Q}} / \mathbb{Q})$ arising from modular forms, Invent. Math. 100 (1990), 431-476. MR 91g:11066

15. J-P. Serre, Sur la topologie des variétés en caractérisque $p$, Symposium Internacional de Topología Algebraica, Universidad Nacional Autónoma de México, 1958, pp. 24-53. MR 20:4559

16. J-P. Serre, Propriétés galoisiennes des points d'order fini des courbes elliptiques, Invent. Math. 15 (1972), 259-331. MR 52:8126

17. G. Shimura, Reduction of algebraic varieties with respect to a discrete valuation ring of the basic field, Amer. J. Math. 77 (1955), 134-176. MR 16:616d

18. G. Shimura, Introduction to the Arithemtic Theory of Automorphic Functions, Iwanami Shoten, Tokyo, and Princeton Univ. Press, Princeton, 1971. MR 47:3318

19. G. Shimura, The special values of the zeta functions associated with Hilbert modular forms, Duke Math. J. 45 (1978), 637-679; 48 (1981), 697. MR 80a:10043; MR 82j:10051

20. J.H. Silverman, The Arithmetic of Elliptic Curves, Graduate Texts in Math., vol. 106, Springer-Verlag, New York, Berlin and Heidelberg, 1986.

21. G. Stevens, Arithmetic on Modular Curves, Progr. in Math., vol. 20, Birkhäuser, Basel, 1982. MR 87b:10050

22. G. Stevens, The cuspidal group and special values of L-functions, Trans. Amer. Math. Soc. 291 (1985), 519-550. MR 87a:11056

23. G. Stevens, Stickelberger elements and modular parametrizations of elliptic curves, Invent. Math. 98 (1989), 75-106. MR 90m:11089 
24. A. Wiles, Modular curves and the class group of $\mathbb{Q}\left(\zeta_{p}\right)$, Invent. Math. 58 (1980), 1-35. MR 82j:12009

25. A. Wiles, On p-adic representations for totally real fields, Ann. of Math. 123 (1986), 407-456. MR 87g: 11142

Department of Mathematics and Statistics, McMaster University, Hamilton, OnTARIO, CANADA L8S 4K1

Current address: Department of Mathematics, The University of Hong Kong, Pokfulam Road, Hong Kong 